\title{
The Action Mechanism of Rotor-Stator Interaction on Hydraulic and Hydroacoustic Characteristics of a Jet Centrifugal Pump Impeller and Performance Improvement
}

\author{
Rong Guo ${ }^{1,2, *}$, Rennian Li ${ }^{1,2, *}$, Renhui Zhang ${ }^{1,2}$ and Wei Han ${ }^{1,2}$ \\ 1 School of Energy and Power Engineering, Lanzhou University of Technology, Lanzhou 730050, China; \\ zhangrh@lut.cn (R.Z.); hanwei@lut.cn (W.H.) \\ 2 Key Laboratory of Fluid Machinery and Systems, Gansu Province, Lanzhou 730050, China \\ * Correspondence: guorong@lut.edu.cn (R.G.); lirn@lut.cn (R.L.); Tel.: +86-093-1297-3750
}

Received: 7 December 2019; Accepted: 6 February 2020; Published: 10 February 2020

\begin{abstract}
The aim of this study was to investigate the action mechanism of the rotor-stator interaction (RSI) on the transient flow field and hydrodynamic noise field inside the impeller of jet centrifugal pumps (JCPs) and optimize effects of the guide vane on the hydraulic and hydroacoustic characteristics of the impeller. The numerical method of CFD (computational fluid dynamics) coupled with CFA (computational fluid acoustics) was used to analyze the correlation between the guide vane and the flow/sound performances of the impeller. The orthogonal test method, with the hydroacoustic performance of the impeller taken as the objective, was used to optimize the structural parameters of the guide vane for the stability of the hydraulic performance of the JCP. The results show that the RSI leads to a significant increase in the hydroacoustic level of the impeller, but it is indispensable for improving the hydraulic performance of the pump. The RSI effect on the fluctuation intensity of the transient flow field inside the impeller is much more sensitive than the time-average, and the fluctuation intensity of the flow field is positively correlated with the vortex intensity inside the impeller. When the impeller geometry is constant, the evolution processes of the flow field inside the impeller are mainly related to the blade number of the guide vane; when the number of guide vanes is given, the RSI effect on the hydroacoustic characteristic of the impeller is characterized by a positive correlation between the total sound pressure level (SPL) and the fluctuation intensity of the flow field. The frequency spectrum characteristics of the hydroacoustic SPL of the impeller are not consistent with the pressure fluctuation characteristics inside the impeller. The pressure fluctuation characteristics are related not only to the blade number and speed of the impeller but also to its wake characteristics determined by the guide vane. The optimization scheme for the stable hydraulic performance of the JCP significantly reduced the total SPL of the impeller compared with the original scheme, which verifies the feasibility of using the weight matrix optimization method to obtain the global optimization scheme.
\end{abstract}

Keywords: jet centrifugal pump; rotor-stator interaction; hydraulic performance; hydroacoustic characteristics; sound pressure level; optimization

\section{Introduction}

The rotor-stator interaction (RSI) is a ubiquitous phenomenon in turbomachinery [1]. It spreads up- and downstream, resulting in the formation of strong flow field fluctuations in the vaneless area between the dynamic and static cascades, which have an important impact on the stability, safety, and robustness of machines. A certain degree of the RSI effect cannot be avoided, but excessive 
RSI effects can reduce the efficiency of the unit, cause fatigue damage of flow passage components, and increase vibration and noise, among other negative outcomes [2,3]. During the past several decades, researchers have used numerical or experimental methods to study the RSI effect on the transient flow field, hydraulic excitation force, vibration, noise, and other characteristics of vane pumps. Dong et al. [4] experimentally studied the effect of modifications to tongue and impeller geometries on the flow structure and the resulting noise in a centrifugal pump, and their results demonstrated that the primary sources of noise were associated with interactions between the nonuniform outflux from the impeller (jet/wake phenomenon) and the tongue. González et al. [5] numerically simulated the dynamic and unsteady flow effects due to the impeller-volute interaction inside a centrifugal pump and showed that the strong effects of the secondary flow were concentrated in radial positions close to the impeller exit. Additionally, pressure fluctuations at the blade passing frequency revealed that the blade tongue interacted with the flow at the impeller outlet plane, and the interaction had clear effects, such as increased fluctuation levels under off-design conditions, limited operation ranges, and increased losses. Guo et al. [6] measured pressure fluctuations and the radial fluid forces induced by the rotor-stator interaction and investigated their relationship, and they demonstrated that both the blade pressure fluctuations and the volute static pressures were circumferentially nonuniform (not axisymmetric) under off-design operating conditions. Rodriguez et al. [7] carried out a theoretical analysis to qualitatively predict and explain the moving blade passing frequency and the relationship between their amplitudes. They presented a way to characterize the vibration in the frequency domain resulting from the RSI. Using a one-dimensional approach, Nicolet et al. [8] modeled, simulated, and analyzed the rotor-stator interaction of a scale model of a Francis pump-turbine and a related test rig and found that the pressure patterns of the diametrical mode in the vaneless gap were dominated by the pressure patterns of the resonance. Zhu et al. [9,10] simulated the flow in the centrifugal pump with different volute casings by using a shear stress transport turbulent model, and their findings indicated that the blade passing frequency dominated the pressure fluctuation, radial hydraulic force, and torque in the impeller, whose fluctuations were periodic. Furthermore, they studied the rotor-stator interaction of three tongues of the centrifugal pump using dynamical subgrid-scale models. Rodriguez et al. [11] studied a $100 \mathrm{MW}$ pump-turbine by making use of pressure measurements and vibrations measured in a guide bearing that rotated with the shaft. From their results, they recommended that the vibration caused by rotation with the shaft be measured to detect RSI characteristics in large hydraulic turbomachinery. Zhou et al. [12] simulated a volute-type centrifugal pump by using LES models to reveal the impeller-volute interaction under rotating stall conditions, and they found that the rotating stall phenomenon occurred when the flow rate was decreased to 0.70 of the nominal flow rate; as the flow rate was further decreased, the area of the stall cells increased. Under the rotating stall condition, the blade passing frequency (i.e., three times the rotation frequency) decreased by $50 \%$ because of alternate stalled and unstalled passages; when the rotating stall occurred, the amplitude of the pressure fluctuation was much higher than that at unstalled points. Zhang et al. [13] studied the pressure pulsation characteristics induced by the rotor-stator interaction in a nuclear reactor coolant pump. At the nominal flow, the flow rate distribution in the guide vanes was relatively uniform, and the amplitude of the pressure pulsation reached the minimum. Furthermore, the vortex shedding or backflow from the impeller blade exit had the same frequency as the pressure pulsation, and that the absolute value of phase differences reflected the vorticity intensity. Chalghoum et al. [14,15] analyzed the rotor-stator interaction (impeller and volute) and its effects on the complex internal flow field within a centrifugal pump for a wide range of operating flow rates, and they found that the pressure distributions around the volute tongue were inversely proportional to the evolution of the relative angle between the blade exit and volute tongue. As a result of the rotor-stator interaction, the amplitude and frequencies of the pressure fluctuations rose from the inlet to the outlet of the impeller for the whole flow range. At the volute tongue region, the pressure amplitude was much larger than that at other regions because of the presence of vortices. 
Scholars have extensively researched the influence of the rotor-stator interaction on the hydraulic performance of hydraulic turbomachinery, but the effect on hydroacoustic performance has rarely been studied. In this study, a typical jet centrifugal pump (JCP) was taken as the study object to investigate the action mechanism of the rotor-stator interaction on its impeller hydraulic and hydroacoustic characteristics and optimize these parameters through multi-objective optimization.

\section{Models and Methods}

\subsection{The Model Pump}

The model JCP had the following parameters: rated flow $Q_{d}=2.5 \mathrm{~m}^{3} / \mathrm{h}$, rated water head $H_{d}=23$ $\mathrm{m}$, rated efficiency $\eta_{d}=20 \%$, rotational speed $n=2850 \mathrm{r} / \mathrm{min}$, shaft passing frequency $S P F=47.5 \mathrm{~Hz}$, blade passing frequency of the impeller $B P F_{I}=285 \mathrm{~Hz}$, and blade passing frequency of the guide vane $B P F_{G}=237.5 \mathrm{~Hz}$. The main geometric parameters of the impeller and guide vane are listed in Table 1. Two- and three-dimensional structure diagrams of the model pump are shown in Figure 1.

Table 1. Parameters for the model jet centrifugal pump.

\begin{tabular}{ccc}
\hline Components & Parameter & Value \\
\hline \multirow{3}{*}{ Impeller } & Inlet diameter $D_{I 1}(\mathrm{~mm})$ & 40 \\
& Outlet diameter $D_{I 2}(\mathrm{~mm})$ & 123 \\
& Blade number $Z_{1}$ & 6 \\
& Blade wrap angle $\varphi_{1}\left({ }^{\circ}\right)$ & 76 \\
& Blade outlet width $b_{2}(\mathrm{~mm})$ & 5.3 \\
\hline \multirow{2}{*}{ Guide vane } & Inlet diameter $D_{G 1}(\mathrm{~mm})$ & 125 \\
& Outlet diameter $D_{G 2}(\mathrm{~mm})$ & 64 \\
& Blade number $Z_{2}$ & 5 \\
\hline
\end{tabular}

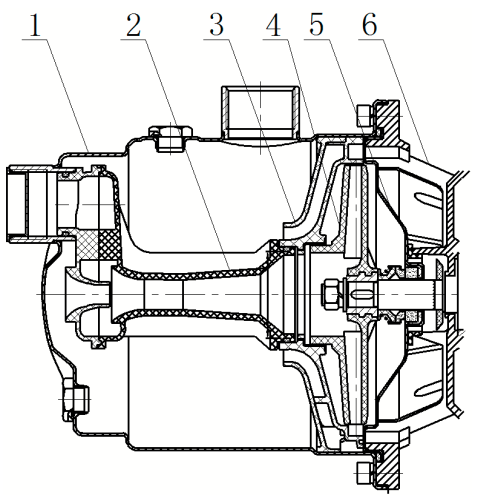

(a)

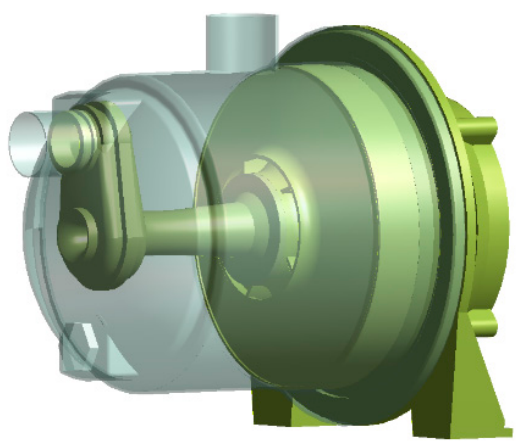

(b)

Figure 1. Structural diagrams of the model pump. (a) Two-dimensional structure diagram; (b) three-dimensional structure diagram. 1: Pump body; 2: Jet; 3: Guide vane; 4: Impeller; 5: Pump cover; 6: Bracket.

\subsection{Research Routes}

The research route followed in this study is shown in Figure 2. The impeller structure remained unchanged while analyzing the correlation between the main geometric parameters of the guide vane and the transient flow field inside the impeller and its induced noise. The hydroacoustic performance of the impeller was taken as the objective, and the guide vane was optimized to increase the stability of the hydraulic performance of the JCP. 


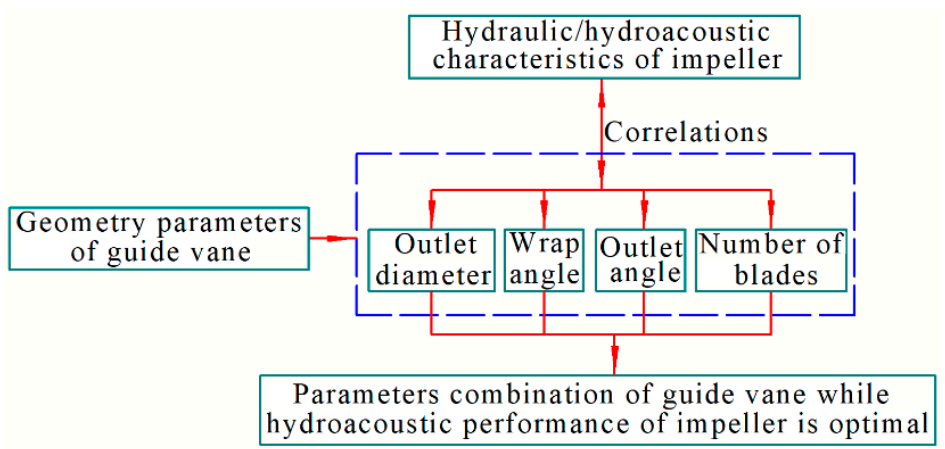

Figure 2. Research routes followed in this study.

\subsection{Method of Computational Fluid Dynamics (CFD)}

\subsubsection{Meshes}

The whole computational domain includes the impeller, guide vane, jet, front pump cavity, back pump cavity, inlet pipe, outlet pipe, and pump body. ANSYS-ICEM (16.0 ANSYS, Inc., Canonsburg, PA, USA) was employed for the discrete computational domain, hexahedral structured meshes were employed for the computational domain of the impeller and guide vane, and tetrahedral unstructured meshes were employed for other flow passage components. The grid independence verification is shown in Table 2, divided into six mesh schemes according to the density of the grid. The grid convergence index (GCI) recommended by ASME is used to estimate the grid dispersion error [16]. GCI is an index with a 95\% confidence interval, which represents the distance between the more dense grid and the progressive value in two comparative grids, and predicts the influence of further grid refinement on the solution. For Schemes 5 and 4 grids, the calculated head uncertainties are $0.19 \%$ and $6.43 \%$, respectively, and efficiency uncertainties are $0.37 \%$ and $7.59 \%$. In order to balance the relationship between computing accuracy and resources, Scheme 4 grid is selected for numerical calculation in this paper. The automatic wall treatment method is used to deal with the boundary layer flow, the method can generate a processing mode that is not sensitive to $\mathrm{Y}^{+}$and allow users to refine or coarsen the near-wall mesh arbitrarily [17]. The impeller and guide vane mesh of the model pump are shown in Figure 3.

Table 2. The grid independence analyzation.

\begin{tabular}{ccccccc}
\hline Schemes & Scheme 1 & Scheme 2 & Scheme 3 & Scheme 4 & Scheme 5 & Scheme 6 \\
\hline Number of the grid & $1,483,718$ & $1,975,598$ & $2,526,764$ & $3,030,389$ & $3,494,674$ & $4,046,652$ \\
Head (m) & 23.97 & 24.22 & 25.43 & 26.15 & 26.17 & 26.16 \\
\hline
\end{tabular}

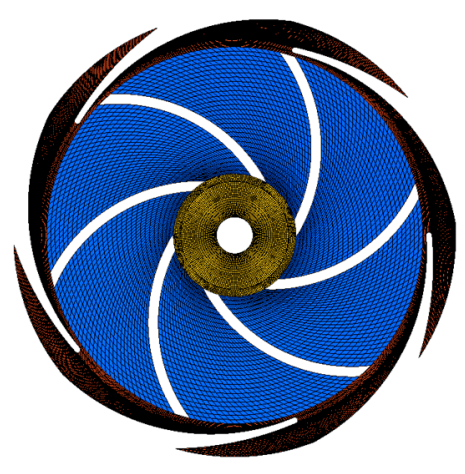

(a) Impeller and positive guide vane

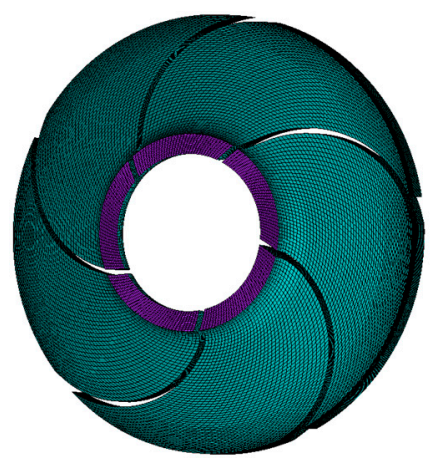

(b) Return guide vane

Figure 3. Grid diagram of the impeller and guide vane of the model jet centrifugal pumps (JCP). 


\subsubsection{Numerical Settings and Boundary Condition}

CFX (16.0, ANSYS Inc., Pittsburgh, PA, USA) was employed for the flow field calculation. The impeller flow fields were calculated in the rotating coordinate system in the multi-coordinate system, and the flow fields of the other components were calculated in the stationary coordinate system. The $k-\omega$ turbulence model was adopted, which is better than the $k-\varepsilon$ model in the aspect of near-wall flow analysis [17], and GGI (general grid interface) technology was employed to exchange data between the rotor and stator. The automatic near-wall treatment method was applied to the flow near the wall. Pressure inlet and velocity outlet boundary conditions were set, and the inlet pressure value was set according to the value obtained from the experiment. All solid walls were characterized as non-slip walls, and roughness was set to $25 \mu \mathrm{m}$ according to actual processing. The governing equations were solved by a second-order upwind formula, and time terms were discretized by a second-order implicit scheme. All residuals were less than $10^{-5}$. The time-step was set to $0.000117 \mathrm{~s}$, which means that the impeller rotated about 2 degrees per time-step. First, steady calculations were carried out, and the results were taken as the initial value for unsteady calculations. Pressure fluctuation information files from 10 rotation cycles on the impeller surface were output for hydroacoustic calculations after the flow field showed a stable periodic change.

\subsubsection{Time-Average and Fluctuation Intensity of the Flow Field}

The unsteady flow characteristics of the model pump were analyzed by a statistical method. The unsteady physical quantities include two parts on each grid node $(x, y, z)$ of the flow domain, the time-averaged component $\bar{\phi}$, and the periodic component $\widetilde{\phi}$, where the periodic component represents the variation in physical quantities in a rotation period of the impeller [18]. The two components are respectively expressed as follows:

$$
\begin{gathered}
\bar{\phi}(n)=\frac{1}{N} \sum_{j=0}^{N-1} \phi\left(n, t_{0}+j \Delta t\right), \\
\widetilde{\phi}(n)=\phi(n, t)-\bar{\phi}(n)
\end{gathered}
$$

where $n, N$, and $t_{0}$ are the grid nodes, the number of samples in an impeller rotation period, and the starting time of the rotation cycle, respectively.

The non-dimensional standard deviation of the periodic component $\widetilde{\phi}$ is defined as the flow field fluctuation intensity, which includes the pressure fluctuation intensity, velocity fluctuation intensity, turbulence energy fluctuation intensity, and so on.

The pressure fluctuation intensity is expressed as

$$
C_{P}^{*}=\frac{\sqrt{\frac{1}{N} \sum_{j=0}^{N-1} \widetilde{P}\left(n, t_{0}+j \Delta t\right)^{2}}}{0.5 \rho U_{2}^{2}} .
$$

The velocity fluctuation intensity is expressed as

$$
C_{U}^{*}=\sqrt{\frac{1}{N} \sum_{j=0}^{N-1} \widetilde{U}\left(n, t_{0}+j \Delta t\right)^{2}} / U_{2} .
$$

where $U_{2}$ is the circumferential speed at the impeller outlet. 


\subsection{Method of Computational Fluid Acoustics (CFA)}

In this paper, LMS Virtual.Lab 12.0 ( Siemens, Berlin, Germany ) and the acoustic finite element method (FEM) were used to solve the hydrodynamic noise of the impeller, and the wide-band calculation method of fan noise was adopted, which did not need a Fourier transform when importing pressure fluctuation data from the impeller surface. The element length of the acoustic FEM mesh was determined by Equation (5):

$$
L \leq \frac{c}{6 f_{\max }}
$$

where $c$ and $f_{\max }$ are the speed of sound and the maximum computational frequency.

Because of the structural characteristics of the model JCP, the length of the mesh element was set to $6 \mathrm{~mm}$. When the hydraulic noise was solved, the surface of other flow passage components was set as the total reflection wall. That is, there was no transmission of sound through the wall, and the sound only traveled upstream and downstream along the water. The boundary conditions of the inlet and outlet of the pump were defined as the property of total sound absorption. The monitoring point was set at the pump outlet pipe at 3 times the pipe-diameter.

For measuring noise, the $1 / 3$ octave analysis method can reflect the spectrum characteristics of the noise source with relatively high accuracy so as to understand the generation mechanism and put forward reduction measures [19]. In this study, the A-weighted method, which is closest to reflecting the subjective feeling of the human ear, was employed to evaluate the hydroacoustic sound pressure level at each frequency. The total sound pressure level (SPL) was obtained by superposing the A-weighted SPL of each center frequency of $1 / 3$ octave bands, which is the energy summation method shown in Equation (6):

$$
L_{P}=10 \lg \sum_{i=1}^{M} 10^{0.1 L_{P i}}
$$

where $L_{P}$ is the total SPL after evaluation and summation, $\mathrm{dBA} ; M$ is the summation number; and $L_{P i}$ is the SPL of each center frequency of $1 / 3$ octave bands after evaluation.

\subsection{Numerical Validation}

Numerical calculations of the flow field and sound field were validated using the JCP test-bed of hydraulic performance and hydrodynamic noise in the Key Laboratory of Fluid Machinery and Systems, Gansu Province. The test system is shown in Figure 4. The position of the hydrophone at the pump outlet is the same as that of the noise monitoring point in the numerical calculation.

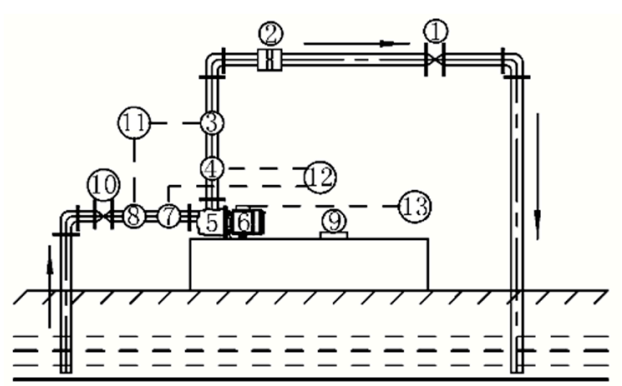

(a)

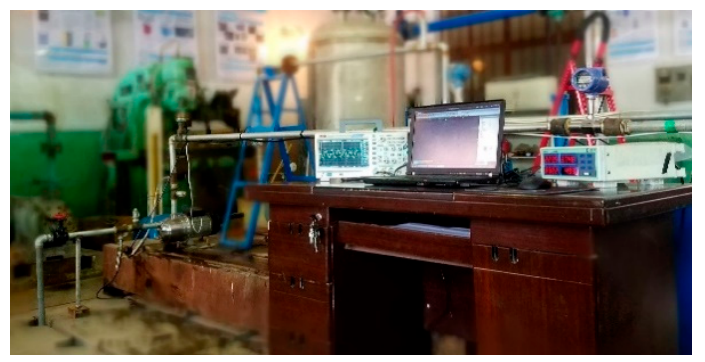

(b)

Figure 4. Test system. (a) Schematic diagram of the test system structure; (b) test site. 1: Valve at pump outlet; 2: Flowmeter; 3: Pressure sensor at pump outlet; 4: Hydrophone at pump outlet; 5: Model pump; 6: Electric motor; 7: Hydrophone at pump inlet; 8: Pressure sensor at pump inlet; 9: Tachometer; 10: Valve at pump inlet; 11: Computer; 12: Oscilloscopes; 13: Measuring instrument of electric power.

Figure 5a compares the curves of hydraulic performance between the experiment and the numerical simulation. The variation trend of the performance curve is consistent between numerical calculations 
and the experiment, and the values at different working points are similar. The maximum error of the water head is $4.6 \%$, and efficiency is $4.1 \%$ : these results confirm the accuracy of the hydraulic calculation results.

In Figure $5 b$, the frequency response curves of the sound pressure level at the monitoring point are compared between numerical calculations and the experiment under rated conditions. As a whole, the level of noise from the experiment is higher than that from the calculation; both the hydroacoustic SPL of the guide vane and that of the impeller have a pronounced amplitude peak at $B P F_{I}$. This is because the JCP contains mechanical noise and hydrodynamic noise that are from different flow passage components and have different characteristics. These different noises can be enhanced by overlapping each other, but they also can be weakened by subtracting from each other. In addition, the flow field calculation cannot completely and accurately capture the flow separation, secondary flow, vortex, or local slight cavitation.

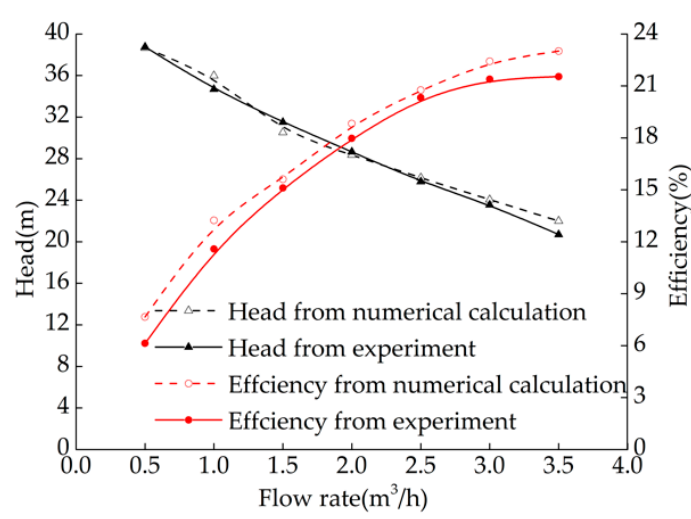

(a)

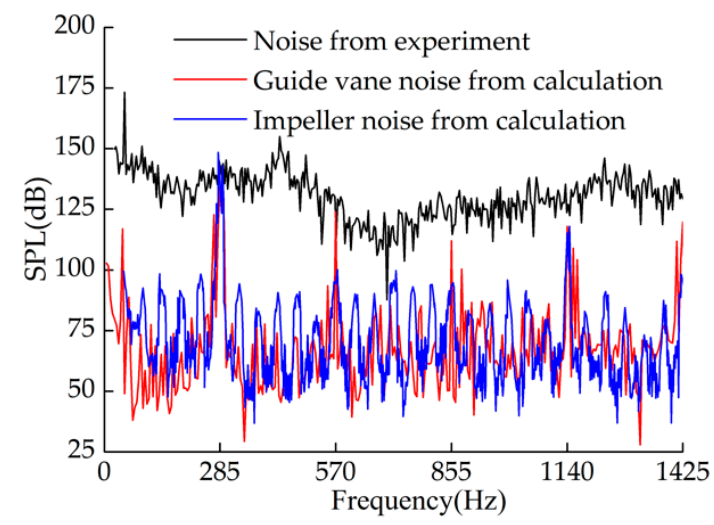

(b)

Figure 5. Comparison of performances between the experiment and numerical calculation. (a) Comparison of hydraulic performance; (b) comparison of frequency response curves of the sound pressure level.

\subsection{Method to Determine the Global Optimization Scheme}

A weight matrix analysis method was employed to obtain the global optimization scheme of a multi-objective orthogonal experimental design. The comprehensive balance method or comprehensive scoring method involves extensive calculations and neglects the differences and connections between indexes, and the weight matrix analysis method overcomes these shortcomings. The influence degree of factors on the index and the primary and secondary order can be assessed by calculating the weights of different levels of factors on the experiment results to quickly reveal the global optimization scheme [20]. The method requires the construction of a three-layer data model with $n$ factors and $m$ levels for each factor according to the data structure, as shown in Table 3.

Table 3. Data structure of the weight matrix analysis method.

\begin{tabular}{ccccc}
\hline Index Layer & \multicolumn{5}{c}{ Indices of Experimental Investigation } \\
\hline Factor Layer & $K_{1}$ & $K_{2}$ & $\ldots$ & $K_{n}$ \\
Level Layer & $K_{11} K_{12} \ldots K_{1 m}$ & $K_{21} K_{22} \ldots K_{2 m}$ & $\ldots$ & $K_{n 1} K_{n 2} \ldots K_{n m}$ \\
\hline
\end{tabular}

In Table 2, the first layer is the index layer of the experimental investigation, and the matrix $\boldsymbol{M}$ (as shown in Equation (7)) is established. The average value of factor $i$ at level $j$ is defined as $k_{i j}$. If a larger index value corresponds to a better value, then $k_{i j}=K_{i j}$; otherwise, $k_{i j}=1 / K_{i j}$. The second layer is the 
factor layer, and the matrix $T$ (as shown in Equation (8)) is established. The third layer is the level layer, and the matrix $\boldsymbol{R}$ (as shown in Equation (9)) is established, where $r_{i}$ is the range of factor $i$.

$$
\begin{aligned}
& \boldsymbol{M}=\left[\begin{array}{cccccccccccc}
K_{11} & K_{12} & \cdots & K_{1 m} & 0 & 0 & \cdots & 0 & 0 & 0 & \cdots & 0 \\
0 & 0 & \cdots & 0 & K_{21} & K_{22} & \cdots & K_{2 m} & 0 & 0 & \cdots & 0 \\
0 & 0 & \cdots & 0 & 0 & 0 & \cdots & 0 & 0 & 0 & \cdots & 0 \\
0 & 0 & \cdots & 0 & 0 & 0 & \cdots & 0 & K_{n 1} & K_{n 2} & \cdots & K_{n m}
\end{array}\right]^{T} \\
& \boldsymbol{T}=\left[\begin{array}{cccc}
T_{1} & 0 & 0 & 0 \\
0 & T_{2} & 0 & 0 \\
\cdots & \cdots & \cdots & \cdots \\
0 & 0 & 0 & T_{m}
\end{array}\right], T_{i}=1 / \sum_{j=1}^{m} K_{i j} \\
& \boldsymbol{R}=\left[\begin{array}{llll}
R_{1} & R_{2} & \cdots & R_{m}
\end{array}\right], R_{i}=r_{i} / \sum_{i=1}^{m} r_{i}
\end{aligned}
$$

The weight matrix $\omega$ affecting the experimental index is defined as

$$
\boldsymbol{\omega}=\boldsymbol{M T R}=\left[\omega_{1}, \omega_{2}, \cdots, \omega_{\mathrm{m}}\right]^{T}
$$

where

$$
\omega_{1}=K_{11} T_{1} R_{1}, K_{11} T_{1}=K_{11} / \sum_{j=1}^{m} K_{1 j}
$$

Because $K_{11} T_{1}$ is the ratio of level 1 to all levels of factor 1 , the $\omega_{1}$ is product of $K_{11} T_{1}$ and $R_{1}$, which reflects the influence degree of level 1 of factor 1 on the index.

\section{Correlations between the Guide Vane and Hydraulic and Hydroacoustic Characteristics of the Impeller}

The values of the main geometric parameters for the original positive guide vane are shown in Table 4. When analyzing the influence of a certain parameter on the hydraulic and hydroacoustic characteristics of the impeller, other parameters should remain unchanged.

Table 4. Main geometric parameters of the original positive guide vane.

\begin{tabular}{ccccc}
\hline Parameters & Blade Number $Z_{2}$ & Wrap Angle $\varphi_{2} /{ }^{\circ}$ & Inlet Angle $\beta_{G 1} /{ }^{\circ}$ & Inlet Diameter $D_{G 1} / \mathbf{m m}$ \\
\hline Value & 5 & 68 & 5 & 125 \\
\hline
\end{tabular}

\subsection{Influence of Guide Vane Blades}

Table 5 shows the correlations between the guide vane and hydraulic and hydroacoustic characteristics of the impeller. As shown in the table, the guide vanes play a very important role in the hydraulic performance of the pump and the hydroacoustic characteristics of the impeller. Without positive and return guide vanes, the water head, hydraulic efficiency, the shaft power of the pump, and the SPL of the impeller are greatly reduced; this also holds if only the positive guide vane removed. 
Table 5. The influence of guide vanes on the hydraulic and hydroacoustic characteristics of the impeller.

\begin{tabular}{cccccccc}
\hline $\begin{array}{c}\text { Guide Vane } \\
\text { Models }\end{array}$ & \multicolumn{2}{c}{ Characteristics of the Impeller } & & \multicolumn{2}{c}{$\begin{array}{c}\text { Performance of the } \\
\text { Pump }\end{array}$} \\
\hline & $\begin{array}{c}\text { Energy Head of } \\
\text { inlet/m }\end{array}$ & $\begin{array}{c}\text { Energy Head of } \\
\text { outlet/m }\end{array}$ & $\begin{array}{c}\text { Energy } \\
\text { increment } / \mathrm{m}\end{array}$ & $\begin{array}{c}\text { Shaft Power } \\
/ \mathrm{W}\end{array}$ & $\begin{array}{c}\text { Total } \\
\text { SPL/dBA }\end{array}$ & $\begin{array}{c}\text { Water } \\
\text { Head/m }\end{array}$ & $\begin{array}{c}\text { Hydraulic } \\
\text { Efficiency } \\
/ \%\end{array}$ \\
\hline I & -1.14 & 27.01 & 28.15 & 426.4 & 92.1 & 5.20 & 8.40 \\
II & 4.66 & 30.42 & 25.76 & 631.0 & 128.4 & 21.23 & 23.17 \\
III & 6.51 & 31.09 & 24.58 & 678.1 & 141.6 & 26.85 & 27.27 \\
\hline
\end{tabular}

I: No positive and return guide vanes; II: No positive guide vanes; III: Prototype.

Figure 6 shows the time-average and the fluctuation intensity curves of pressure and velocity on the blade surface of the impeller under rated condition for three models of the guide vane. ( $l$ is dimensionless radius from a point on the middle streamline of blade surface to the center of axis, the same applies below). Whether the guide vane is present has little influence on the change rules of the time-average values of pressure and velocity inside the impeller, but it has a very significant influence on their fluctuation intensity. For the model lacking both positive and return guide vanes, the RSI effect disappears completely, and the fluctuation intensity of the flow field on the impeller blade surface is very small, with almost no change along the radius. For the model lacking positive guide vanes only, the RSI effect is considerably weakened, and the fluctuation intensity of pressure and velocity significantly drops relative to the prototype.

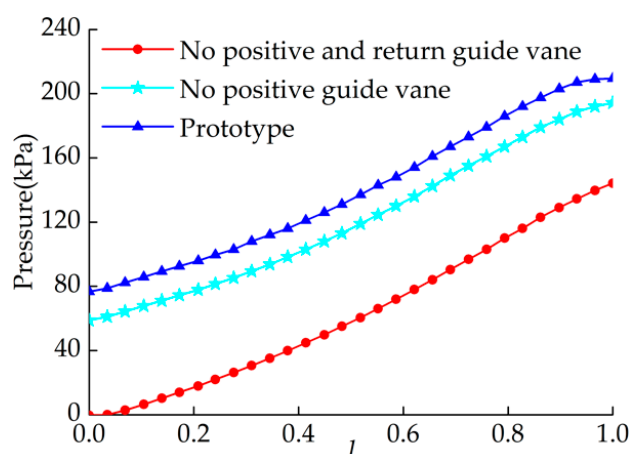

(a)

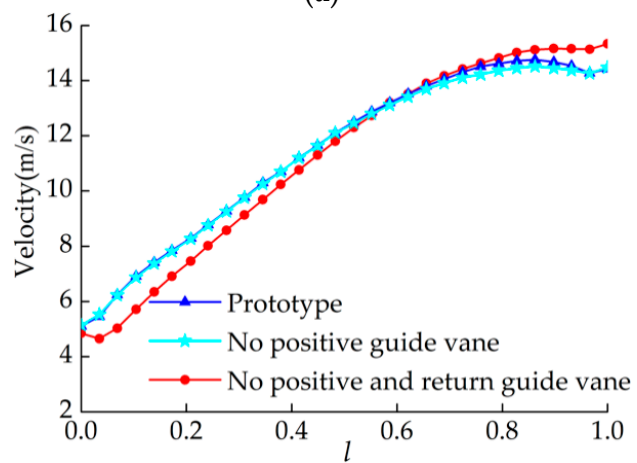

(c)

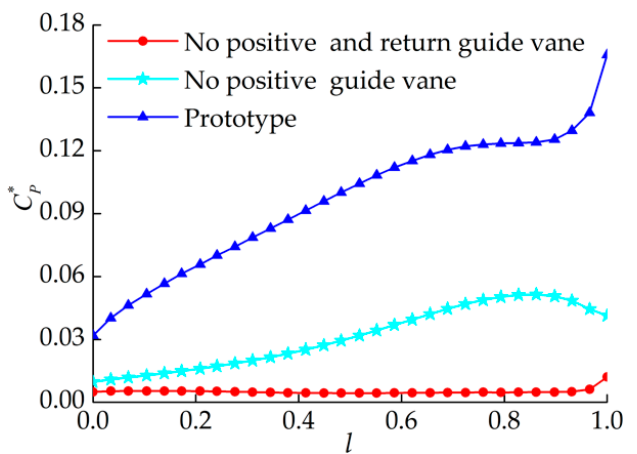

(b)

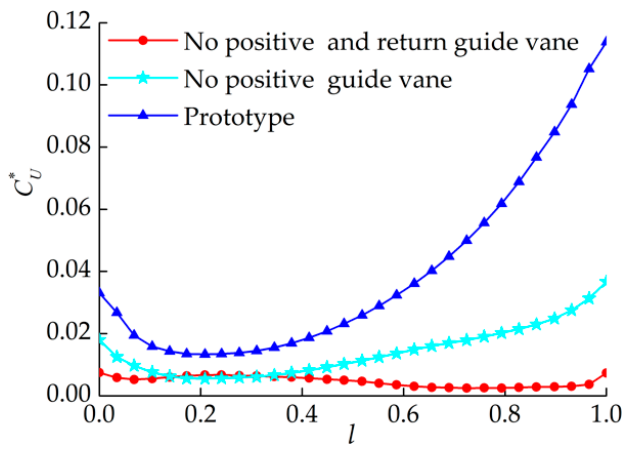

(d)

Figure 6. The time-average and fluctuation intensity curves of pressure and velocity on the blade surface of the impeller with different blade numbers of the guide vane. (a) The curves of the pressure time-average; (b) the curves of pressure fluctuation intensity; (c) the curves of the velocity time-average; (d) the curves of velocity fluctuation intensity.

Figure 7 shows the time (in one impeller rotation cycle; the same applies below) and frequency response curves of pressure inside the impeller for three models of guide vanes. Compared with the other two models, the pressure fluctuation in the time and frequency domains can be almost negligible without positive and return guide vanes. For the models without the positive guide vane, 
the fluctuation characteristics are similar to those of the original model: five waveforms in one cycle, and the dominant frequency is $B P F_{G}$, but the amplitudes are not very prominent at the low-order frequency multiplication of $B P F_{G}$. The above analysis results show that the flow field fluctuation characteristics inside the impeller are closely related to the blade number of the guide vane.

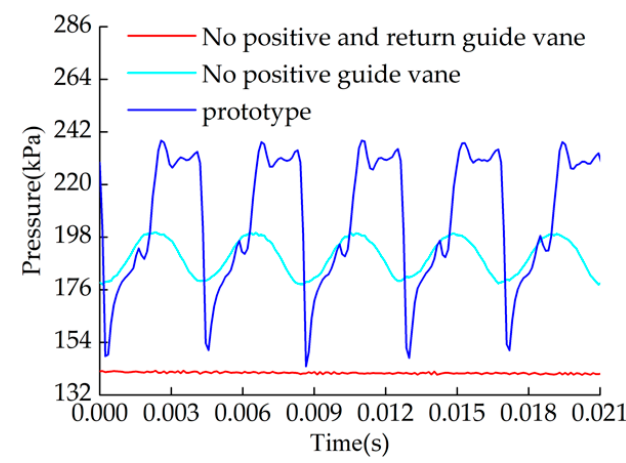

(a) Time domain

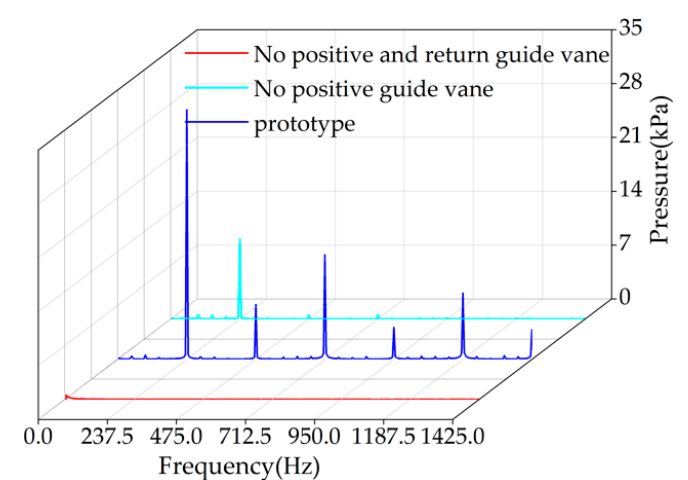

(b) Frequency domain

Figure 7. Time and frequency response curves of pressure inside the impeller for different blade numbers of the guide vane.

Figure 8 shows the frequency response curves of the hydroacoustic SPL of the impeller for three models of the guide vane. All of the curves have periodic fluctuations with the SPF. When the model only lacks positive guide vanes, the curve has large amplitudes at $B P F_{I}$; however, when the model has both positive and return guide vanes, the curve has relatively large amplitudes at $B P F_{I}$ and its frequency multiplication. A comparison with Figure 7 reveals that the frequency spectrum characteristics of the impeller hydroacoustic SPL are not consistent with the pressure fluctuation characteristics inside the impeller.

Figure 9 shows the evolution processes (the processes of the impeller rotating $60^{\circ}$; the same applies below) of the flow field inside the impeller and guide vane for three models. For the model without positive and return guide vanes, the evolution process of vortices inside the impeller is much different from that for the other two models. When the impeller is rotating, the vortex shapes change little in a single flow channel and distribute symmetrically in six channels. For the model without positive guide vanes, the variation law of the transient flow field is similar to that for the prototype, but the strength and size are smaller. For the prototype, the presence of the positive guide vane causes substantial channel blockage in the interface area of the dynamic and static cascades. By combining these results with Figure 8, we find that the frequency spectrum characteristics of the hydroacoustic SPL of the impeller are related not only to the blade number and speed of the impeller but also to its wake characteristics determined by the guide vane.

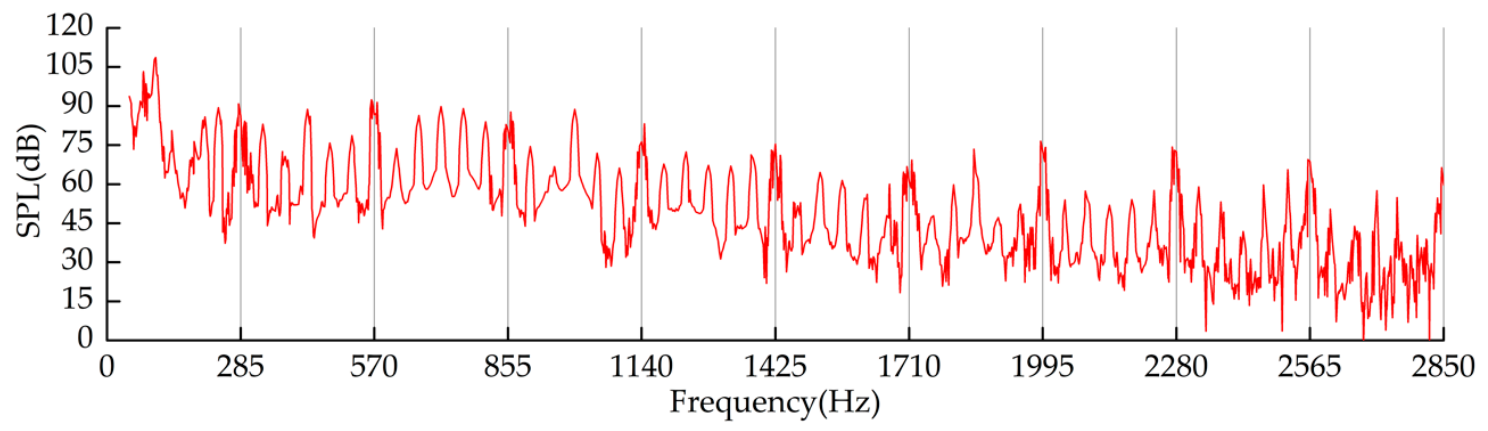

(a) No positive and return guide vanes

Figure 8. Cont. 


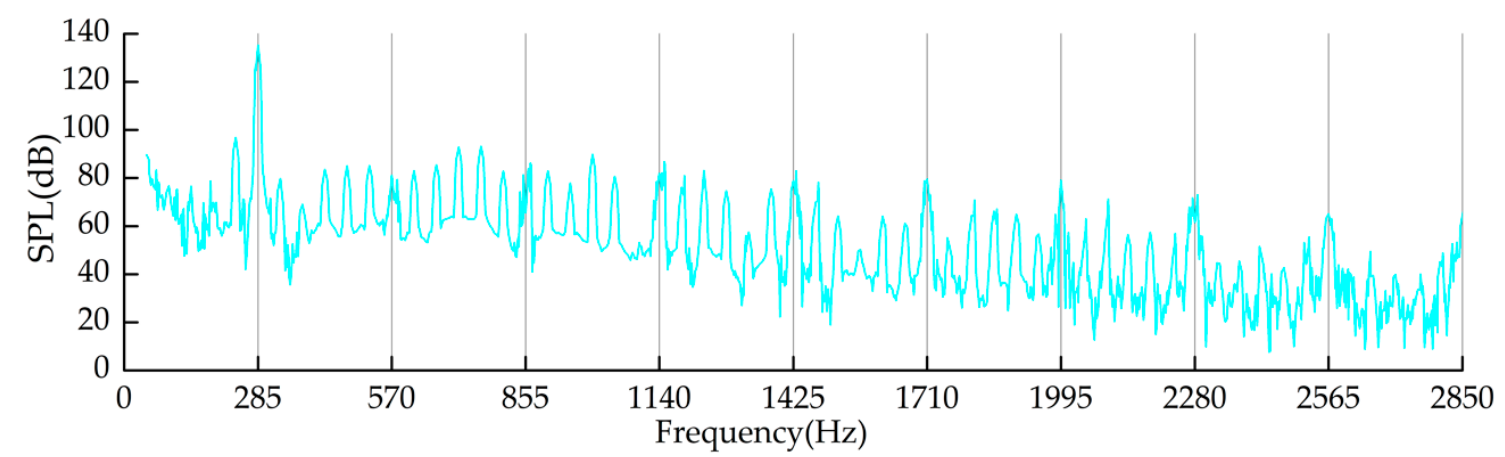

(b) No positive guide vanes

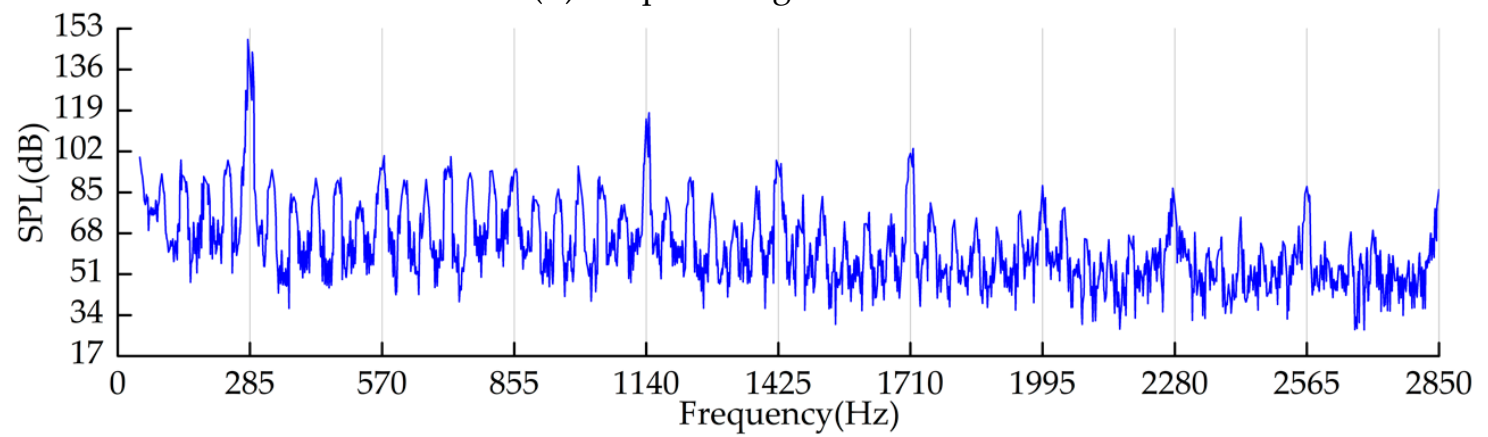

(c) Prototype

Figure 8. Frequency response curves of the hydroacoustic sound pressure level (SPL) of the impeller with different blade numbers of the guide vane.

The analysis results in Figures 6-9 show that the RSI effect leads to a significant increase in the hydroacoustic SPL of the impeller, but it is indispensable for improving the flow field inside the impeller and the hydraulic performance of the pump.
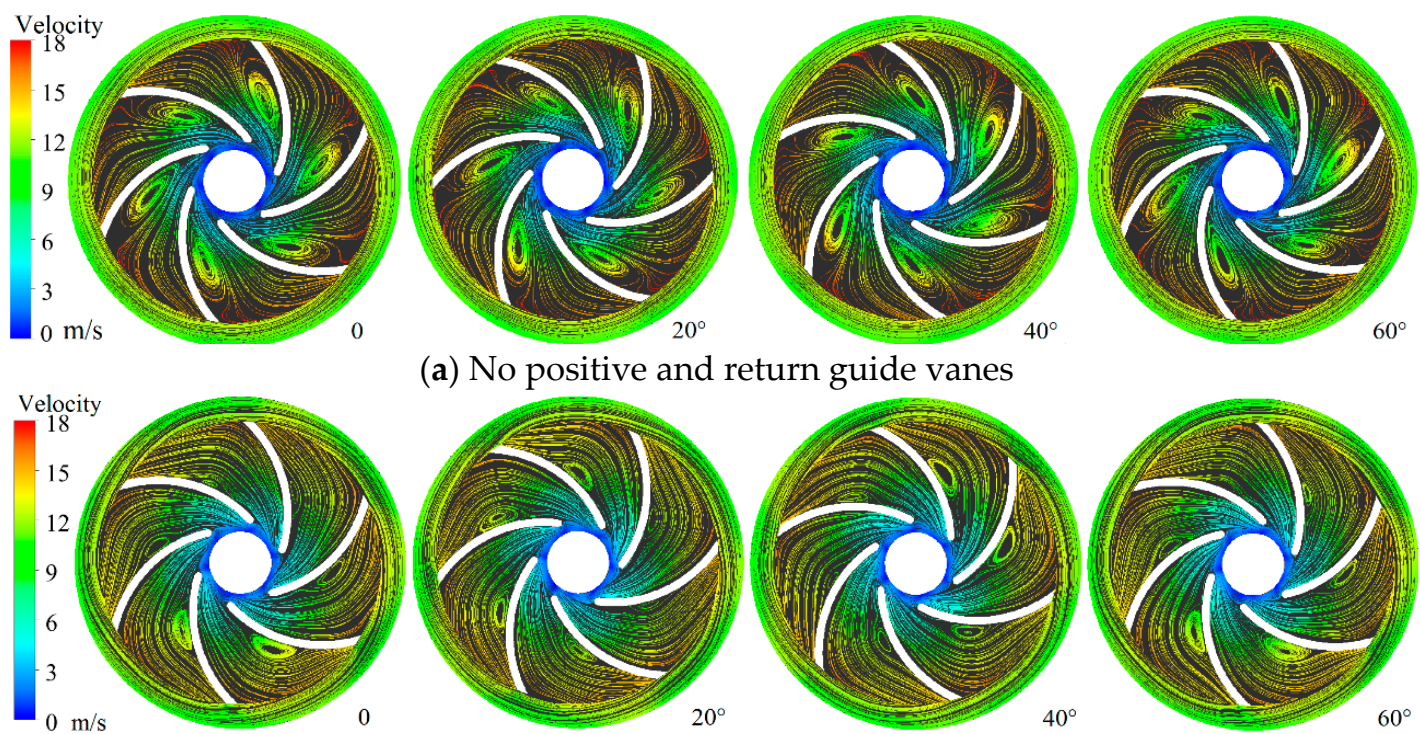

(a) No positive and return guide vanes
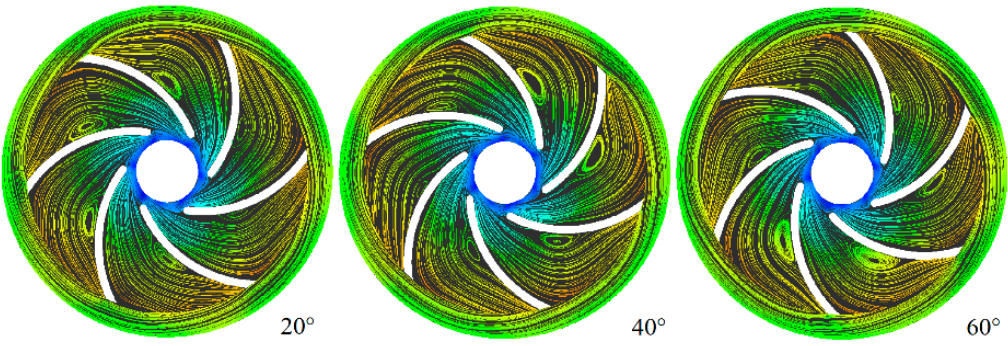

(b) No positive guide vanes

Figure 9. Cont. 

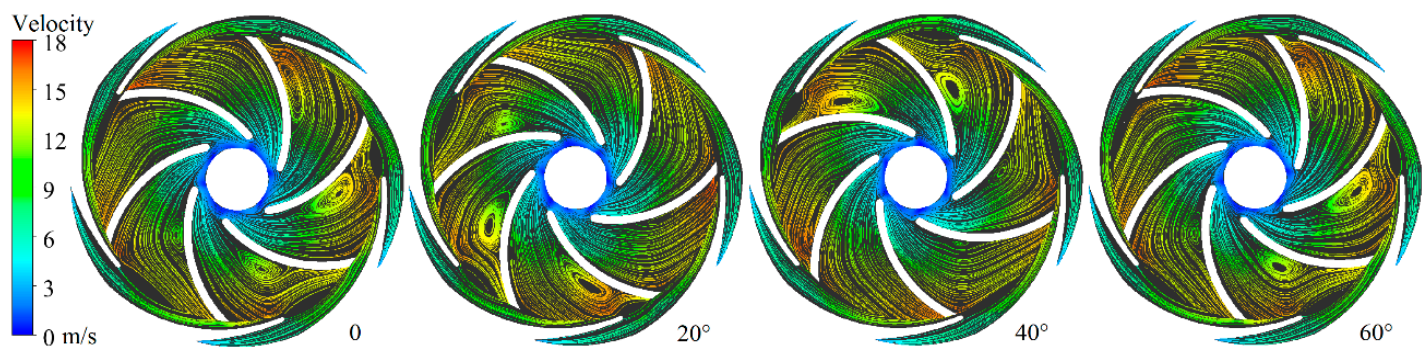

(c) Prototype

Figure 9. Evolution processes of the transient flow field inside the impeller and guide vane with different blade numbers of the guide vane.

\subsection{Blade Number of the Guide Vane}

Table 6 shows the correlations between the number of guide vanes and the hydraulic and hydroacoustic characteristics of the impeller. The data show that as the blade number of the guide vane increases, the energy head of the impeller outlet and the shaft power increase monotonously, while the total SPL of the impeller and the hydraulic performance of the pump first increase and then decrease.

Table 6. Correlations between the number of guide vanes and hydraulic and hydroacoustic characteristics of the impeller.

\begin{tabular}{cccccccc}
\hline $\begin{array}{c}\text { Guide Vane } \\
\text { Parameter }\end{array}$ & \multicolumn{3}{c}{ Characteristics of the Impeller } & \multicolumn{2}{c}{$\begin{array}{c}\text { Performance of the } \\
\text { Pump }\end{array}$} \\
\hline $\begin{array}{c}\text { Blade number } \\
Z_{2}\end{array}$ & $\begin{array}{c}\text { Energy head of } \\
\text { inlet/m }\end{array}$ & $\begin{array}{c}\text { Energy head of } \\
\text { outlet/m }\end{array}$ & $\begin{array}{c}\text { Energy } \\
\text { increment/m }\end{array}$ & $\begin{array}{c}\text { Shaft } \\
\text { power/W }\end{array}$ & $\begin{array}{c}\text { Total } \\
\text { SPL/dBA }\end{array}$ & $\begin{array}{c}\text { Water } \\
\text { head/m }\end{array}$ & $\begin{array}{c}\text { Hydraulic } \\
\text { efficiency/\% }\end{array}$ \\
\hline 3 & 4.10 & 28.86 & 24.76 & 622.4 & 136.4 & 19.76 & 21.87 \\
5 & 6.51 & 31.09 & 24.58 & 678.1 & 141.6 & 26.85 & 27.27 \\
7 & 6.18 & 31.27 & 25.09 & 685.2 & 140.7 & 25.78 & 25.91 \\
\hline
\end{tabular}

Figure 10 shows the time-average and the fluctuation intensity curves of pressure and velocity on the blade surface of the impeller with different numbers of guide vanes. As shown in the figures, as $Z_{2}$ changes, the time-average curves of both the pressure and velocity rise linearly by a similar law along the increasing radius. The time-average curve of pressure is lower when $Z_{2}$ is 3 , which is consistent with the results in Table 5 . The fluctuation intensity of both pressure and velocity are affected very significantly by $Z_{2}$ and drastically decline as it increases.

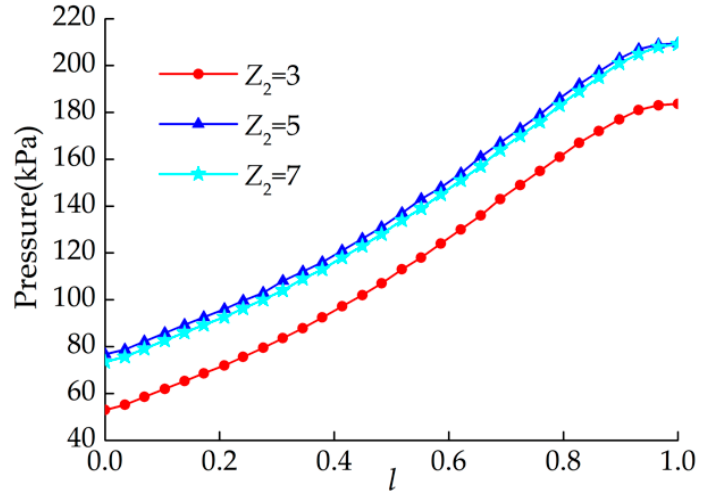

(a)

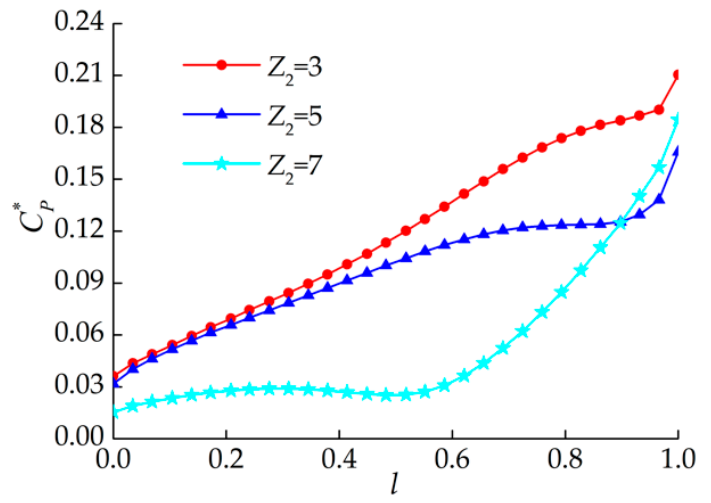

(b)

Figure 10. Cont. 


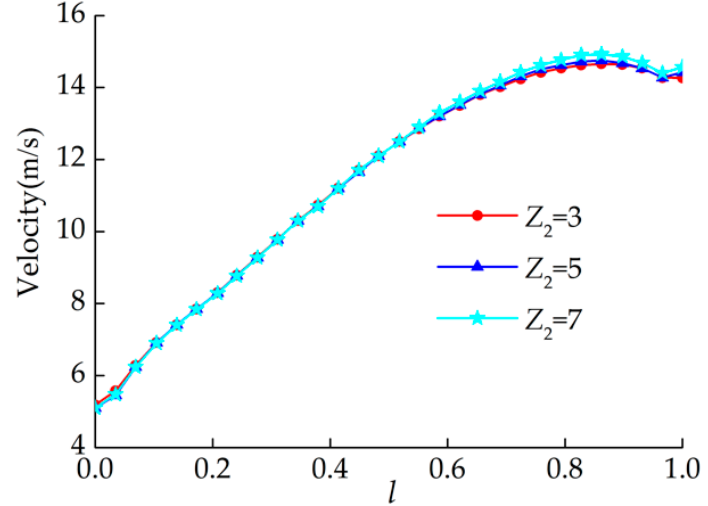

(c)

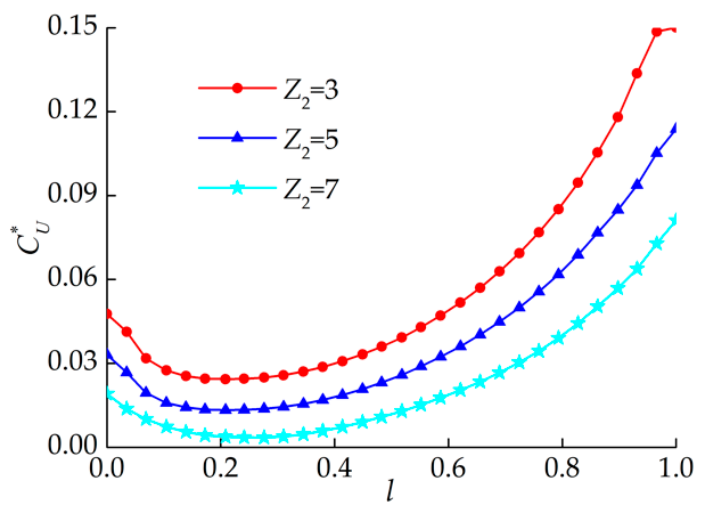

(d)

Figure 10. The time-average and the fluctuation intensity curves of pressure and velocity on the blade surface of the impeller with different numbers of guide vanes. (a) The curves of the pressure time-average; (b) the curves of pressure fluctuation intensity; (c) the curves of the velocity time-average; (d) the curves of velocity fluctuation intensity.

Figure 11 shows the time and frequency response curves of pressure inside the impeller with different numbers of guide vanes. The results show that the number of similar waveforms in a cycle is the same as the number of guide vanes; the frequency curves have large amplitudes at their own $\mathrm{BPF}_{\mathrm{G}}$ and low-order frequency multiplications, but the variation rules are not very consistent, and the positions of the dominant and secondary frequencies are different.

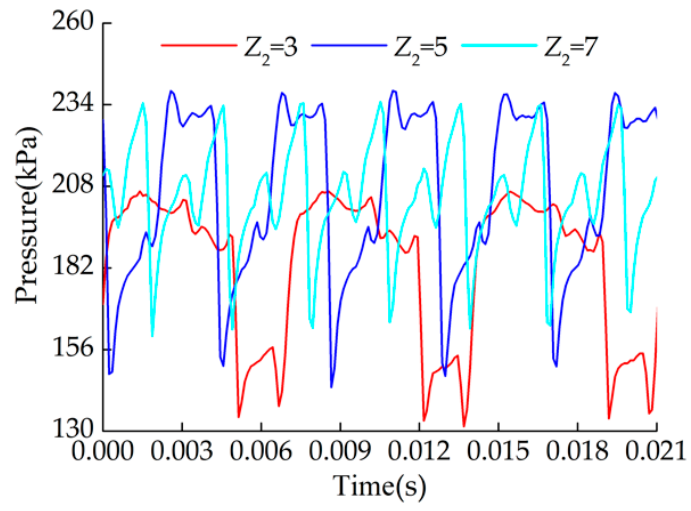

(a) Time domain

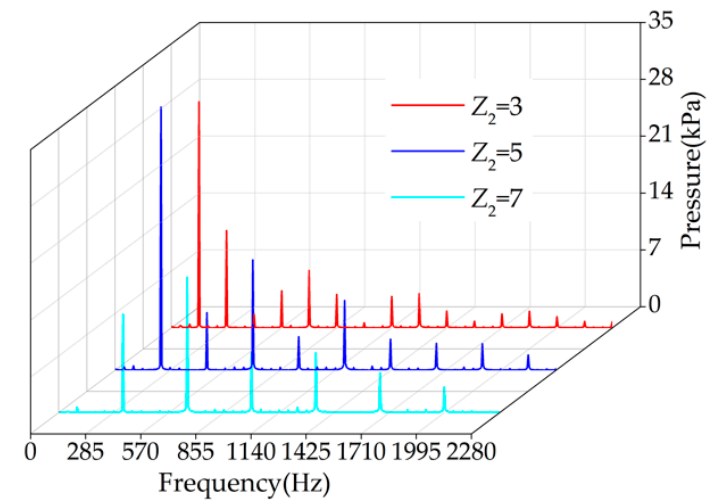

(b) Frequency domain

Figure 11. Time and frequency response curves of pressure inside the impeller with different numbers of guide vanes.

Figure 12 shows the frequency response curves of the hydroacoustic SPL of the impeller with different numbers of guide vanes. The frequency spectrum characteristic of the SPL is similar for different numbers of guide vanes, and all of their dominant frequencies are $B P F_{I}$. 


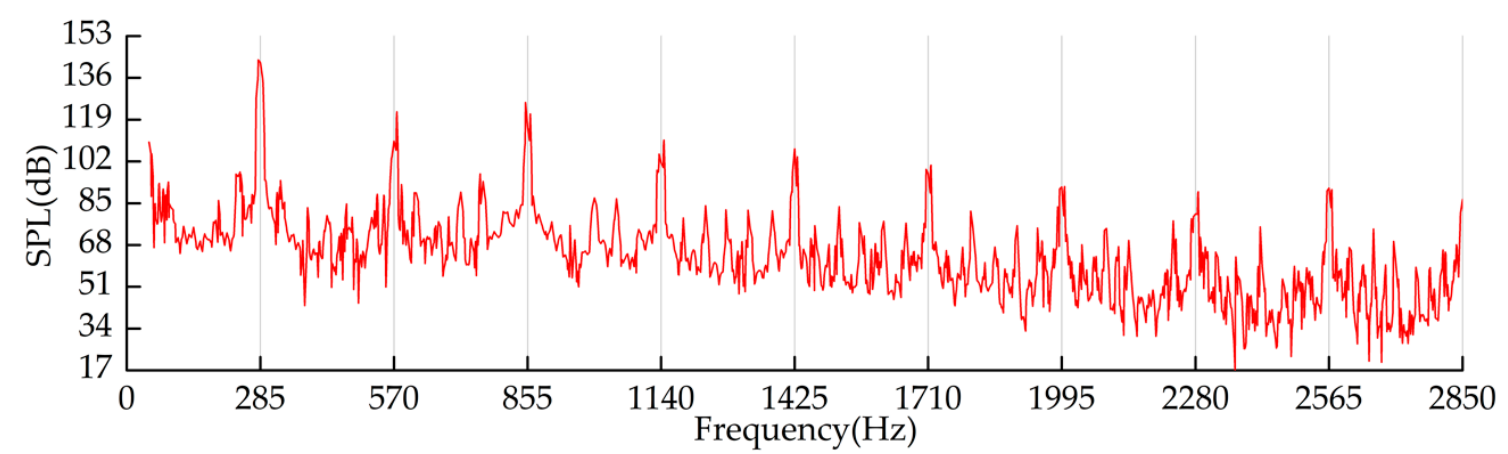

(a) $Z_{2}=3$

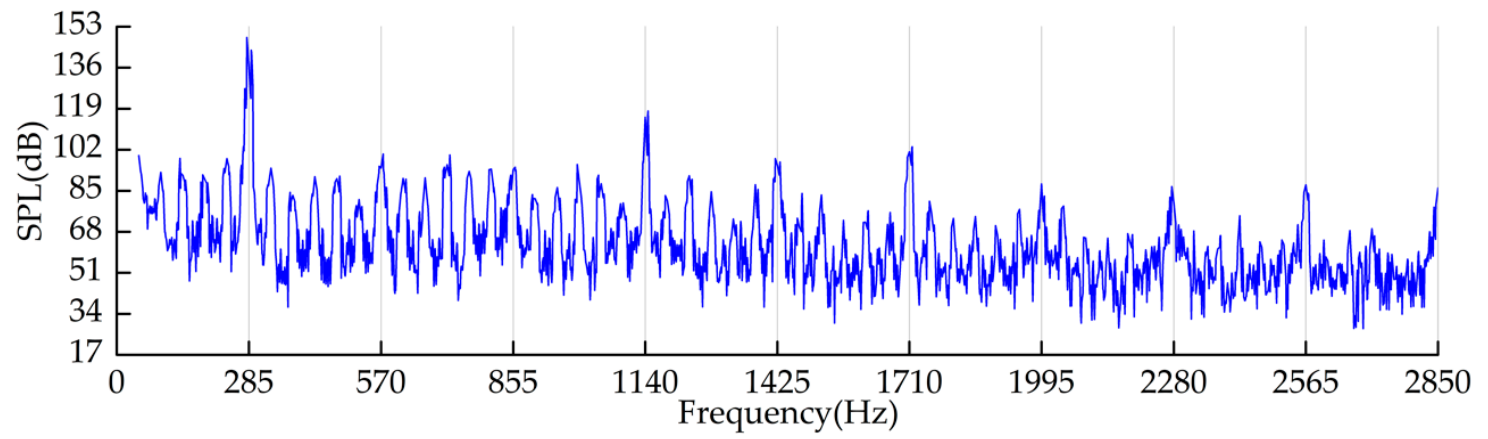

(b) $Z_{2}=5$

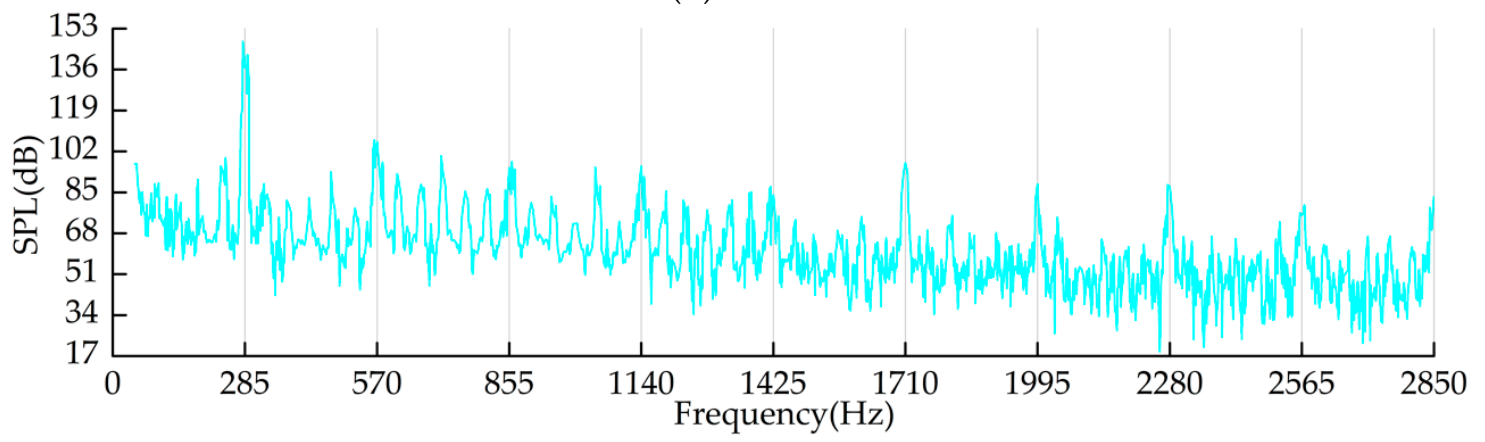

(c) $Z_{2}=7$

Figure 12. Frequency response curves of the hydroacoustic SPL inside the impeller with different numbers of guide vanes.

Figure 13 shows the evolution processes of the flow field inside the impeller and guide vane with different numbers of guide vanes. The evolution rules of the flow field are observed to have obvious differences when $Z_{2}$ is different. The scale of vortices inside the impeller and the degree of flow blockage at the inlet of the guide vane are the most pronounced when $Z_{2}$ is 3 , which results in extremely serious hydraulic losses. Thus, the hydraulic performance of the pump is much lower in this scenario than in the other two schemes in Table 5. With the increase in $Z_{2}$, the strength of the vortices inside the impeller weaken gradually. A comparison with Figure 10 shows a positive correlation between the fluctuation intensity of the transient flow field on the blade surface and the intensity of the vortices inside the impeller: that is, the greater the intensity of the vortices inside the impeller, the greater the fluctuation intensity of the pressure and velocity on the blade surface. 

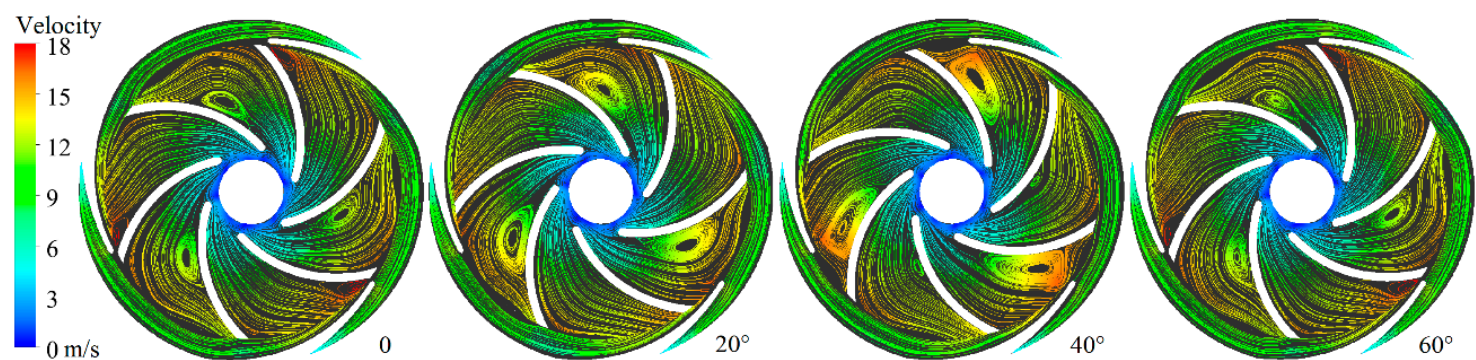

(a) $Z_{2}=3$
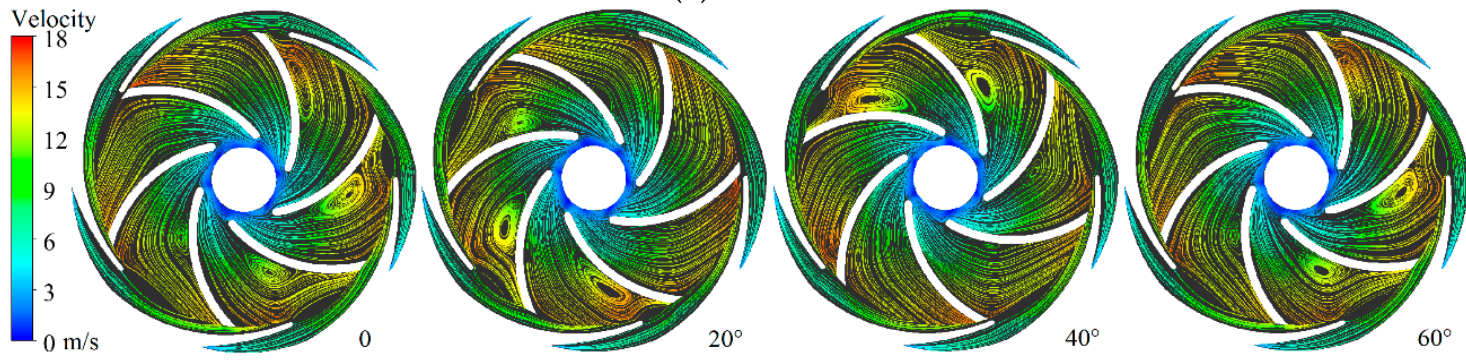

(b) $Z_{2}=5$
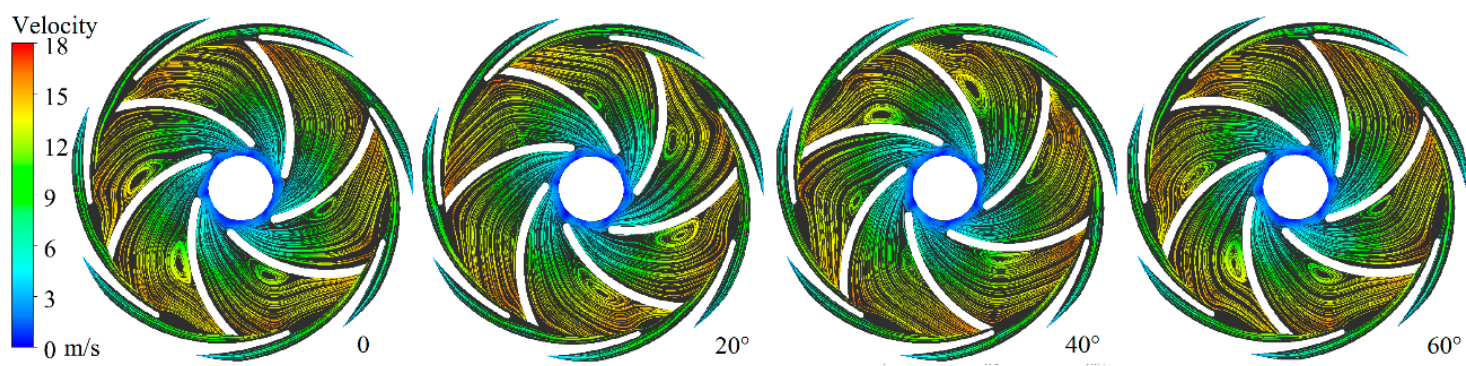

(c) $Z_{2}=7$

Figure 13. Evolution processes of the transient flow field inside the impeller and guide vane with the different blade numbers of the guide vane.

\subsection{Wrap Angle of the Positive Guide Vane}

Table 7 shows the correlations between the wrap angle of the positive guide vane and the hydraulic and hydroacoustic characteristics of the impeller. The data show that as the wrap angle increases, the energy increment and the shaft power of the impeller show a monotonous increasing trend. The total SPL decreases gradually, and the hydraulic performance of the pump first increases and then decreases. That is because the bigger wrap angle and the longer blade can guide the flow more continuously than shorter ones. Means, the less turbulences loss will occur and the performances should be higher; however, if the blades are too long, the blade roughness will cause more friction loss, and the efficiency will decrease again.

Table 7. Correlations between the wrap angle of the positive guide vane and the hydraulic and hydroacoustic characteristics of the impeller.

\begin{tabular}{|c|c|c|c|c|c|c|c|}
\hline \multirow{2}{*}{$\begin{array}{c}\begin{array}{c}\text { Guide Vane } \\
\text { Parameter }\end{array} \\
\text { Wrap angle } \varphi_{2} \\
\left(^{\circ}\right)\end{array}$} & \multicolumn{5}{|c|}{ Characteristics of the Impeller } & \multicolumn{2}{|c|}{$\begin{array}{l}\text { Performance of the } \\
\text { Pump }\end{array}$} \\
\hline & $\begin{array}{c}\text { Energy head of } \\
\text { inlet } / \mathrm{m}\end{array}$ & $\begin{array}{c}\text { Energy head of } \\
\text { outlet } / \mathrm{m}\end{array}$ & $\begin{array}{c}\text { Energy } \\
\text { increment } / \mathrm{m}\end{array}$ & $\begin{array}{c}\text { Shaft } \\
\text { power/W }\end{array}$ & $\begin{array}{c}\text { Total } \\
\text { SPL/dBA }\end{array}$ & $\begin{array}{c}\text { Water } \\
\text { head } / \mathrm{m}\end{array}$ & $\begin{array}{l}\text { Hydraulic } \\
\text { efficiency } / \%\end{array}$ \\
\hline 52 & 6.02 & 30.03 & 24.01 & 670.2 & 146.9 & 25.43 & 26.13 \\
\hline 68 & 6.51 & 31.09 & 24.58 & 678.1 & 141.6 & 26.85 & 27.27 \\
\hline 84 & 6.32 & 31.52 & 25.20 & 698.4 & 139.8 & 26.25 & 25.89 \\
\hline
\end{tabular}

Figure 14 shows the time-average and the fluctuation intensity curves of pressure and velocity on the blade surface of the impeller with different wrap angles of the guide vane. The time-average curves 
of the pressure and velocity are highly similar along the radius with different $\varphi_{2}$, but the curves of fluctuation intensity are affected very significantly and decline drastically as the wrap angle increases.

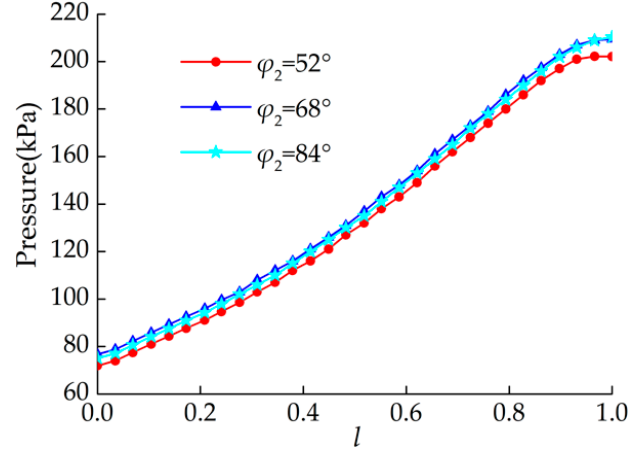

(a)

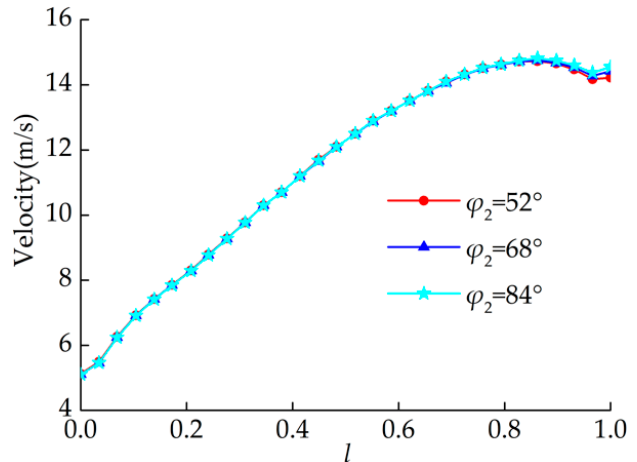

(c)

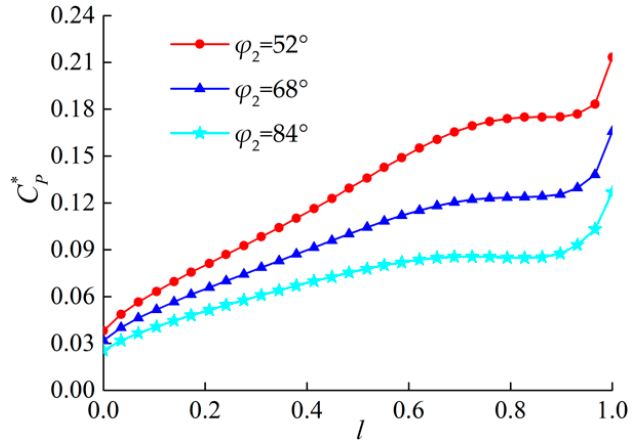

(b)

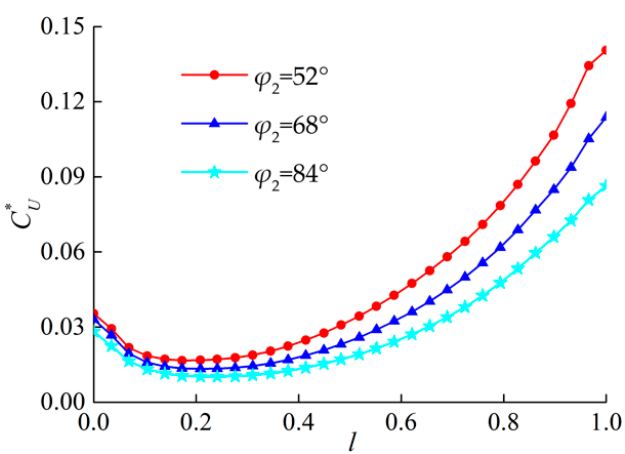

(d)

Figure 14. The time-average and the fluctuation intensity curves of pressure and velocity on the blade surface of the impeller with different wrap angles of the positive guide vane. (a) The curves of the pressure time-average; (b) the curves of pressure fluctuation intensity; (c) the curves of the velocity time-average; (d) the curves of velocity fluctuation intensity.

Figure 15 shows the time and frequency response curves of pressure inside the impeller with different wrap angles of the guide vane. As can be seen from the figure, the variation rules of the pressure in the time and frequency domains are consistent, and the fluctuation amplitude decreases gradually as the wrap angle increases at the dominant and secondary frequencies.

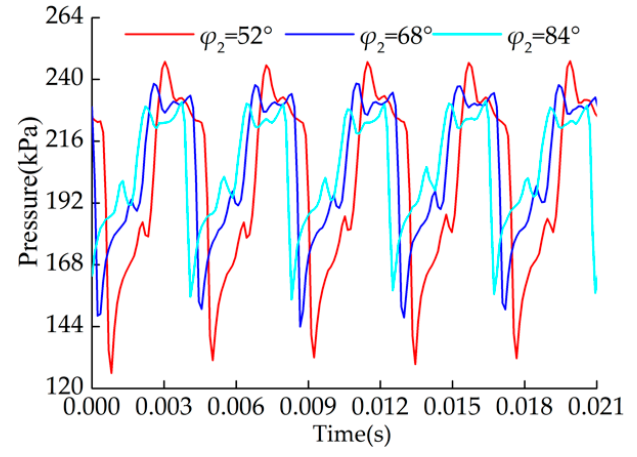

(a) Time domain

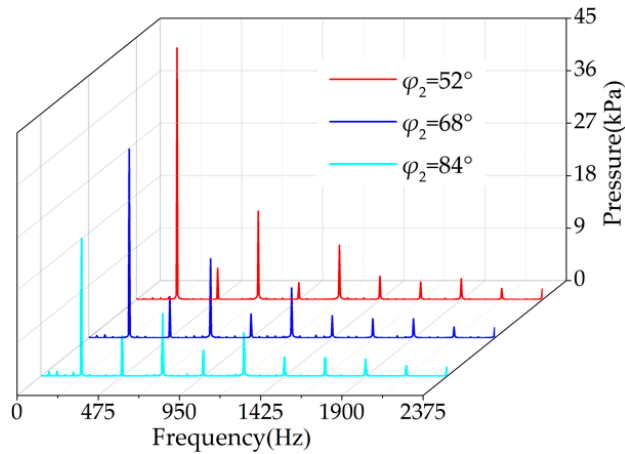

(b) Frequency domain

Figure 15. Frequency response curves of pressure inside the impeller with different wrap angles of the positive guide vane.

Figure 16 shows the frequency response curves of the hydroacoustic SPL of the impeller with different wrap angles of the guide vane. The frequency spectrum characteristic of the SPL are similar 
when the wrap angles are different, and the dominant frequencies for all of them are $B P F_{I}$. The SPL of the model with $\varphi_{2}=52^{\circ}$ is large and decreases as $\varphi_{2}$ increases.

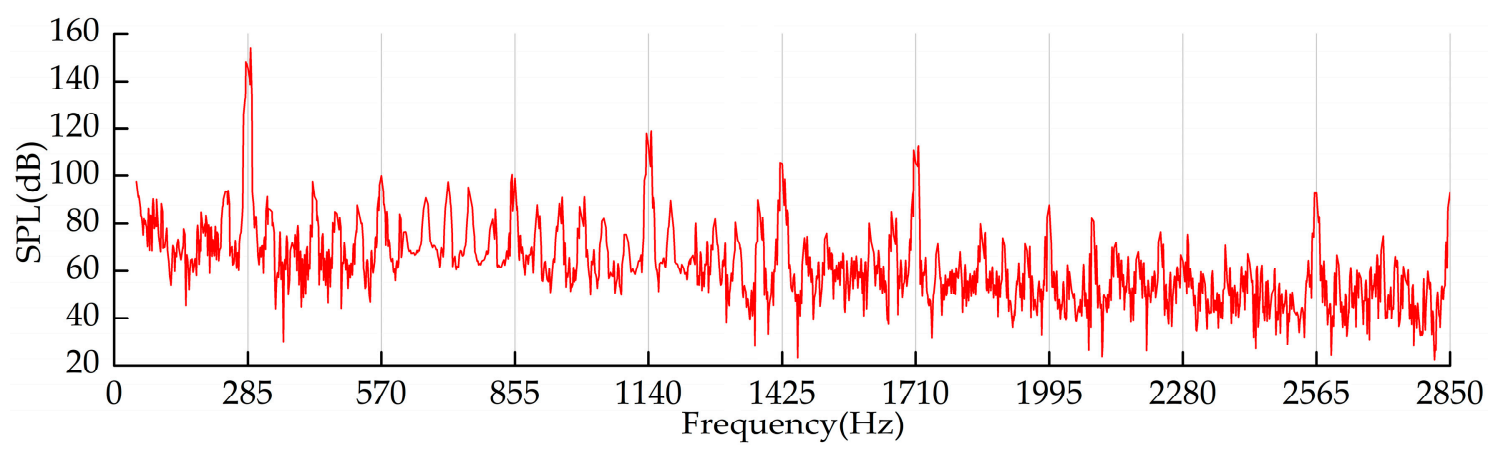

(a) $\varphi_{2}=52^{\circ}$

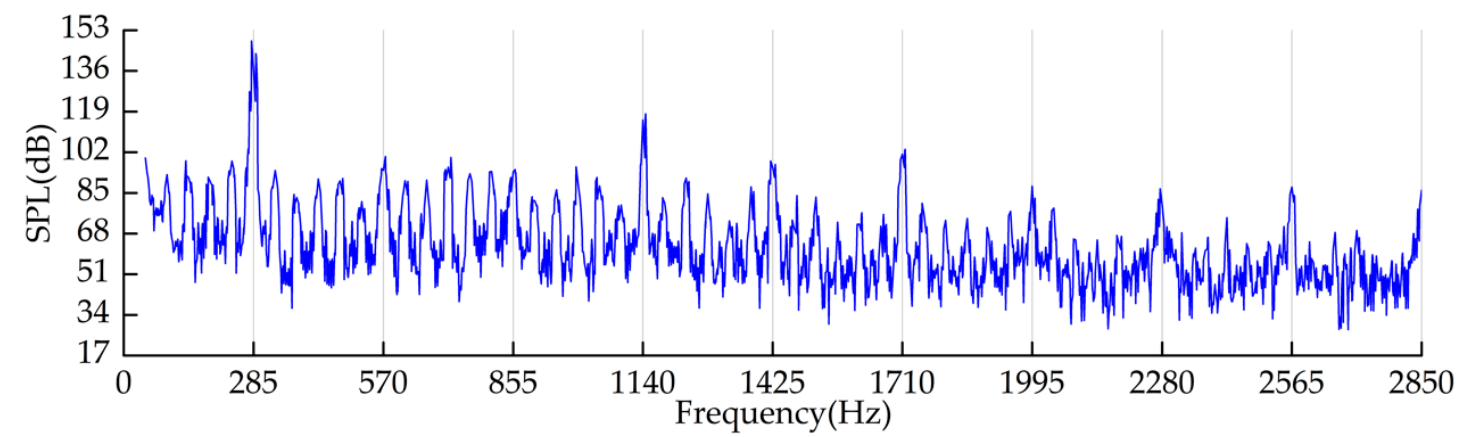

(b) $\varphi_{2}=68^{\circ}$

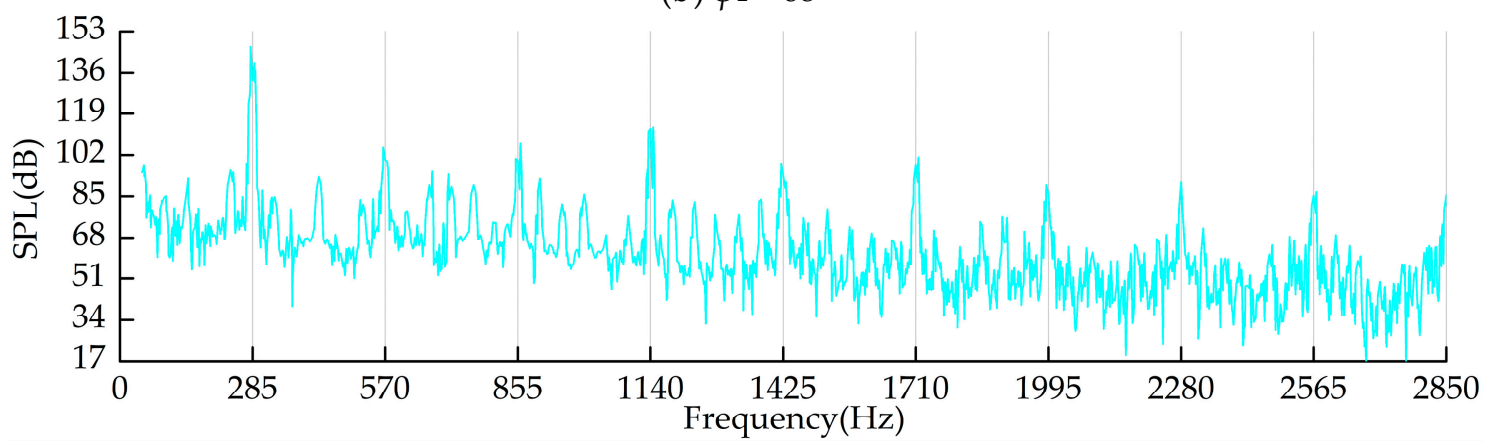

(c) $\varphi_{2}=84^{\circ}$

Figure 16. Frequency response curves of the hydroacoustic SPL inside the impeller with different wrap angles of the positive guide vane.

Figure 17 shows the evolution processes of the flow field inside the impeller and guide vane with different wrap angles of the guide vane; the evolution rules of the flow field are basically the same in the dynamic and static cascades. With the increase in wrap angles, the size and intensity of vortices inside the impeller and the degree of flow blockage at the inlet of the guide vane decrease gradually. This is because when the wrap angle is larger, the change gradient of the blade angle is smaller, the ability of the blades to control the flow is stronger, and the flow inside the guide vane is smoother, resulting in a better fluid transition between the dynamic and static cascades. 

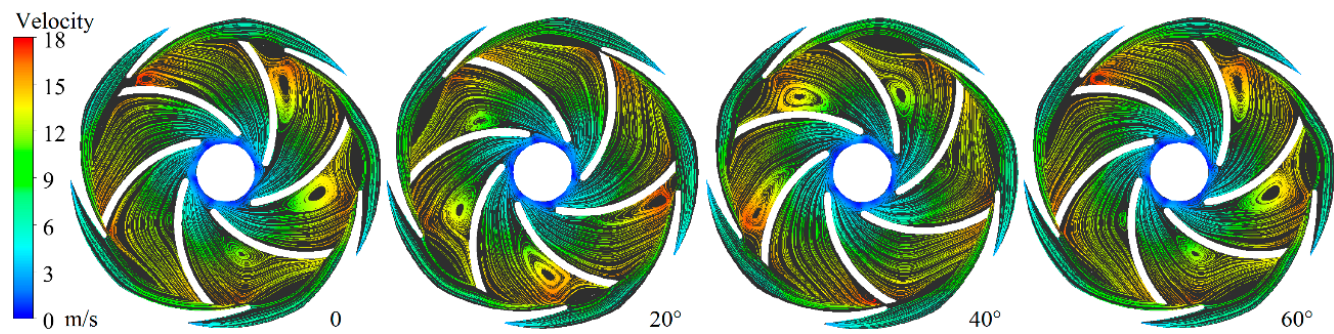

(a) $\varphi_{2}=52^{\circ}$
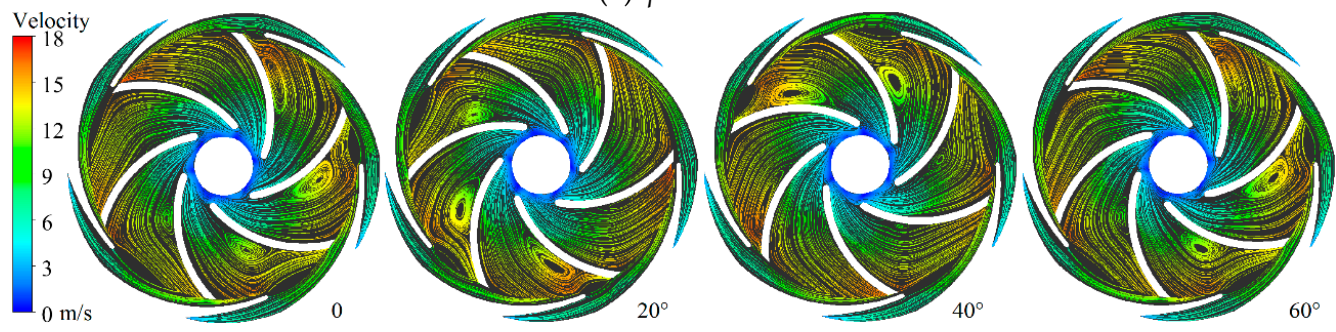

(b) $\varphi_{2}=68^{\circ}$
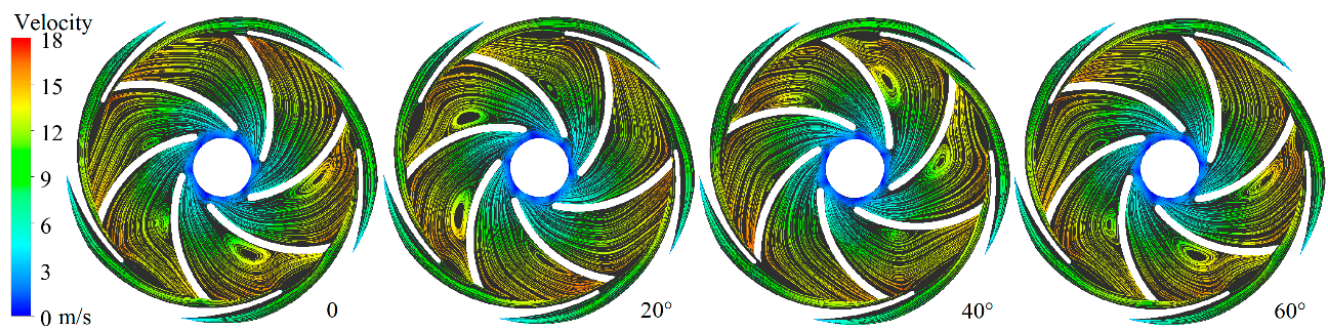

(c) $\varphi_{2}=84^{\circ}$

Figure 17. Evolution processes of the transient flow field inside the impeller and guide vane with different wrap angles of the positive guide vane.

\subsection{Inlet Angle of Guide Vanes}

Table 8 shows the correlations between the inlet angle of the positive guide vane and the hydraulic and hydroacoustic characteristics of the impeller. With the increase in the inlet angle, the energy increment of the impeller shows a monotonous increasing trend, while the hydraulic performance of the pump and the total SPL of the impeller decline gradually, and the shaft power changes little.

Table 8. Correlations between the inlet angle of the positive guide vane and the hydraulic and hydroacoustic characteristics of the impeller.

\begin{tabular}{cccccccc}
\hline $\begin{array}{c}\text { Guide Vane } \\
\text { Parameter }\end{array}$ & \multicolumn{3}{c}{ Characteristics of the Impeller } & & \multicolumn{2}{c}{$\begin{array}{c}\text { Performance of the } \\
\text { Pump }\end{array}$} \\
\hline Inlet angle $\beta_{G 1} /{ }^{\circ}$ & $\begin{array}{c}\text { Energy head of } \\
\text { inlet } / \mathrm{m}\end{array}$ & $\begin{array}{c}\text { Energy head of } \\
\text { outlet } / \mathrm{m}\end{array}$ & $\begin{array}{c}\text { Energy } \\
\text { increment } / \mathrm{m}\end{array}$ & $\begin{array}{c}\text { Shaft } \\
\text { power/W }\end{array}$ & $\begin{array}{c}\text { Total } \\
\text { SPL/dBA }\end{array}$ & $\begin{array}{c}\text { Water } \\
\text { head/m }\end{array}$ & $\begin{array}{c}\text { Hydraulic } \\
\text { efficiency/\% }\end{array}$ \\
\hline 5 & 6.51 & 31.09 & 24.58 & 678.1 & 141.6 & 26.85 & 27.27 \\
10 & 6.14 & 30.89 & 24.75 & 676.2 & 138.7 & 25.73 & 26.21 \\
15 & 5.87 & 30.85 & 24.98 & 676.1 & 136.5 & 24.86 & 25.33 \\
\hline
\end{tabular}

Figure 18 shows the time-average and the fluctuation intensity curves of pressure and velocity on the blade surface of the impeller with different inlet angles of the positive guide vane. Although the inlet angles of the guide vane do not have a very significant effect on the time-average and fluctuation intensity of pressure and velocity, the fluctuation intensity is still more sensitive than the time-average. With the increase in $\beta_{G 1}$, the curves of the fluctuation intensity of the velocity show an overall decline, and the fluctuation intensity of the pressure first decreases and then increases near the impeller outlet. 


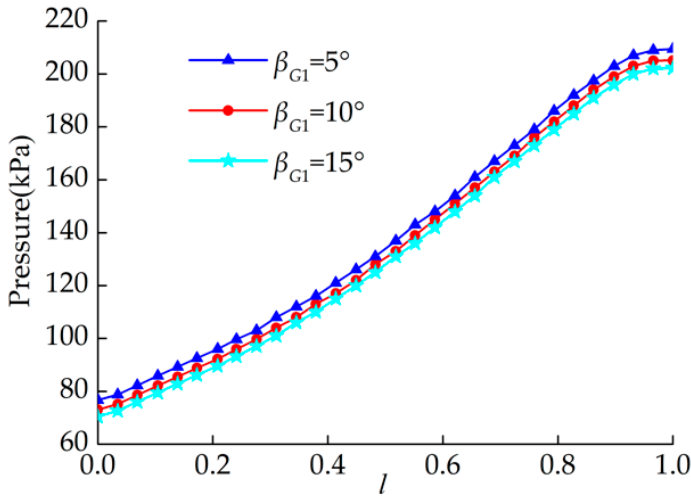

(a)

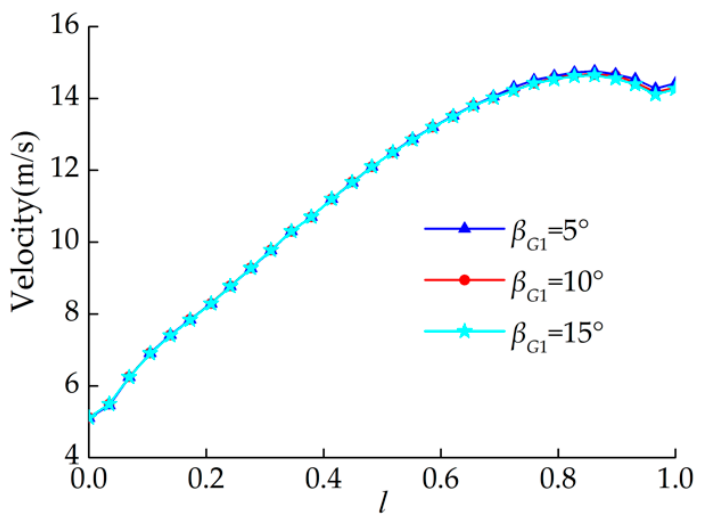

(c)

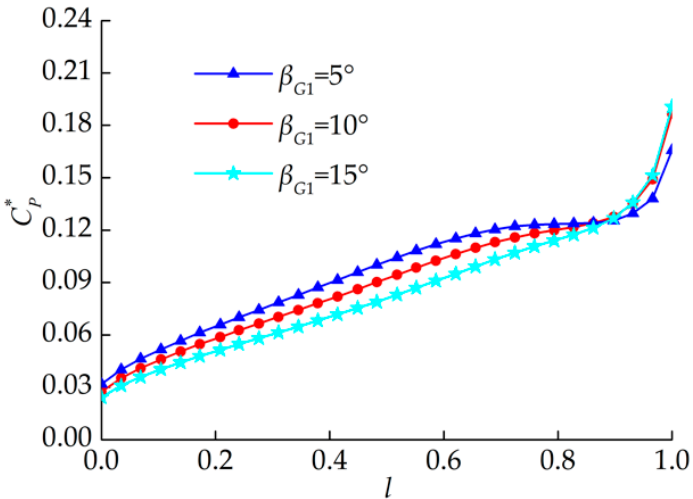

(b)

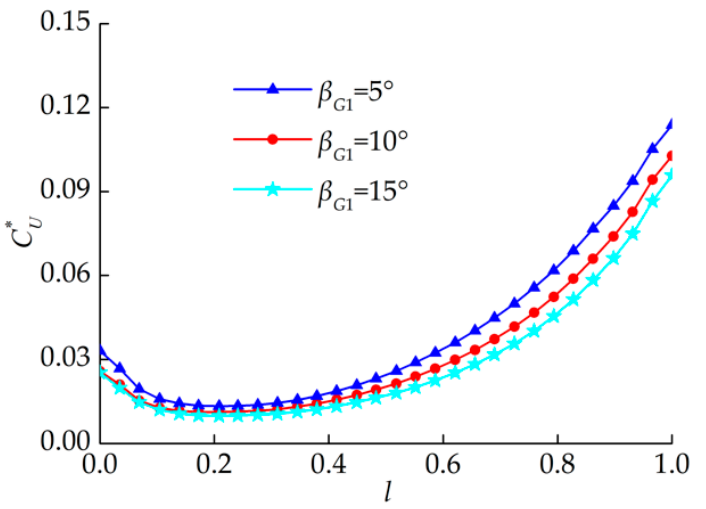

(d)

Figure 18. The time-average and the fluctuation intensity curves of pressure and velocity on the blade surface of the impeller with different wrap angles of the positive guide vane. (a) The curves of the pressure time-average; (b) the curves of pressure fluctuation intensity; (c) the curves of the velocity time-average; (d) the curves of velocity fluctuation intensity.

Figure 19 shows the time and frequency response curves of pressure inside the impeller with different inlet angles of the positive guide vane. As illustrated in the figure, the variation rules of pressure in both the time and frequency domains are identical for different $\beta_{G 1}$, and the fluctuation amplitude decreases gradually as $\beta_{G 1}$ increases at each characteristic frequency.

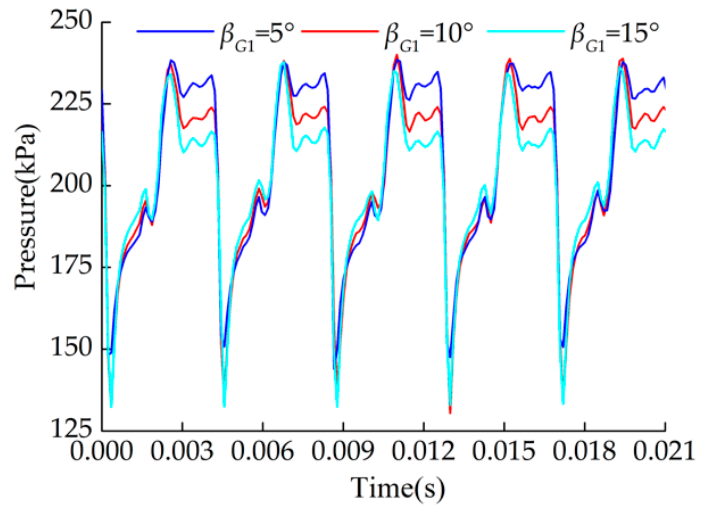

(a) Time domain

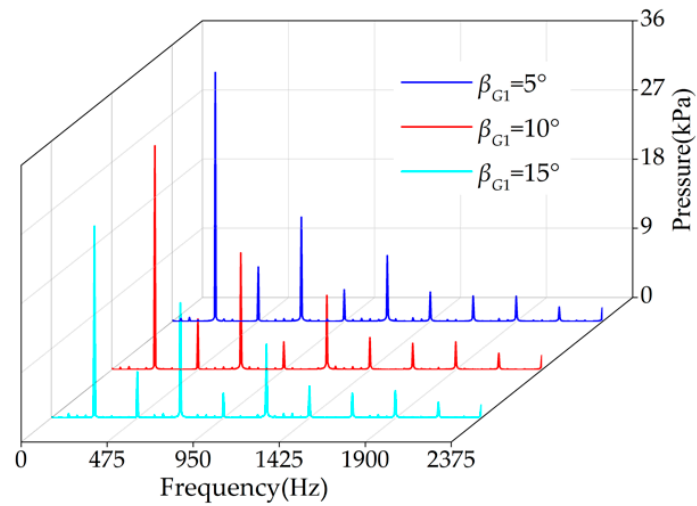

(b) Frequency domain

Figure 19. Time and frequency response curves of pressure inside the impeller with different inlet angles of the positive guide vane. 
Figure 20 shows the frequency response curves of the hydroacoustic SPL of the impeller with different inlet angles of the guide vane. The frequency spectrum characteristic of the SPL is similar for different inlet angles; the dominant frequencies of all of them are $B P F_{I}$, and the SPL decreases as the inlet angle increases.

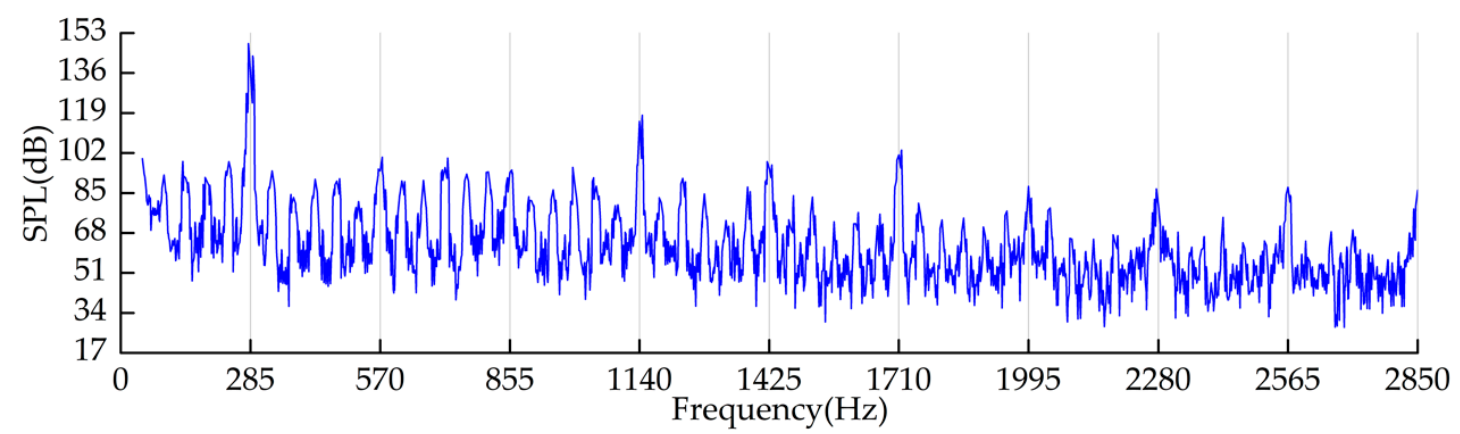

(a) $\beta_{G 1}=5^{\circ}$

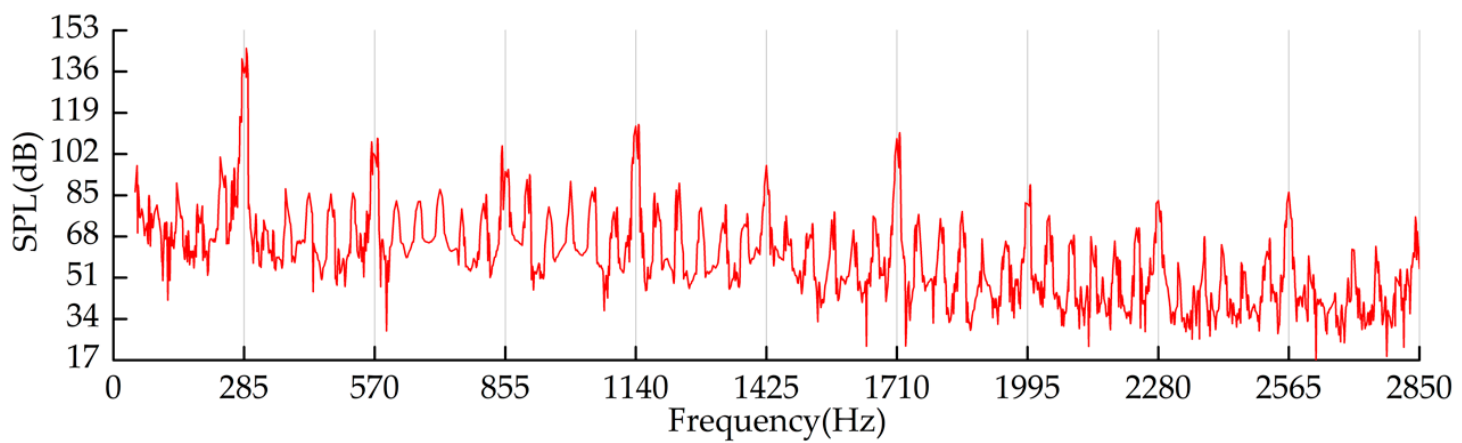

(b) $\beta_{G 1}=10^{\circ}$

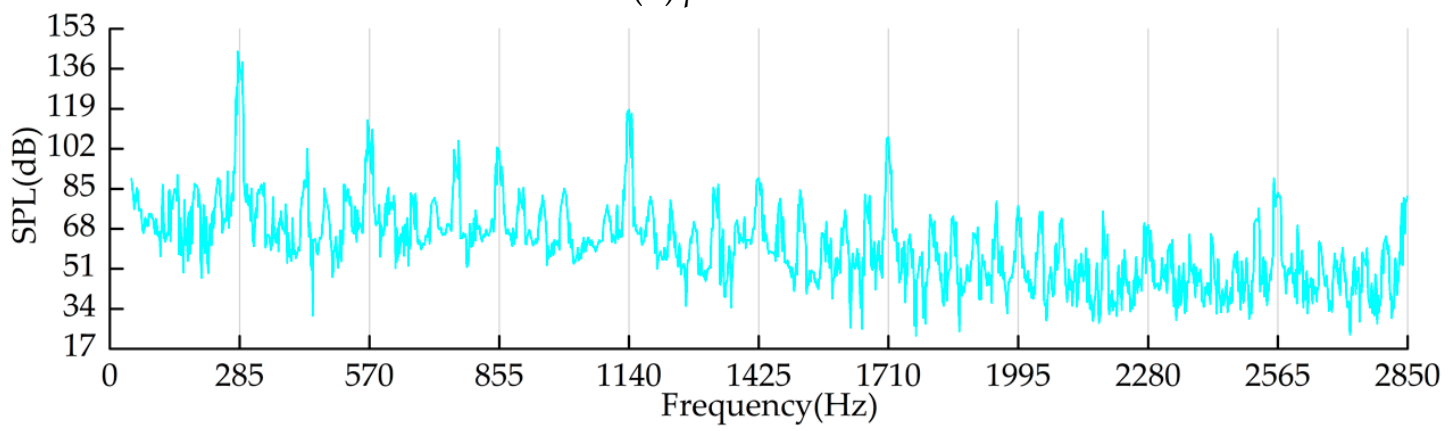

(c) $\beta_{G 1}=15^{\circ}$

Figure 20. Frequency response curves of the SPL inside the impeller with different inlet angles of the positive guide vane.

Figure 21 shows the evolution processes of the flow field inside the impeller and guide vane with different inlet angles of the positive guide vane. The evolution rules of the flow field are basically the same in the dynamic and static cascades, and the differences among the three different models are not very large. 

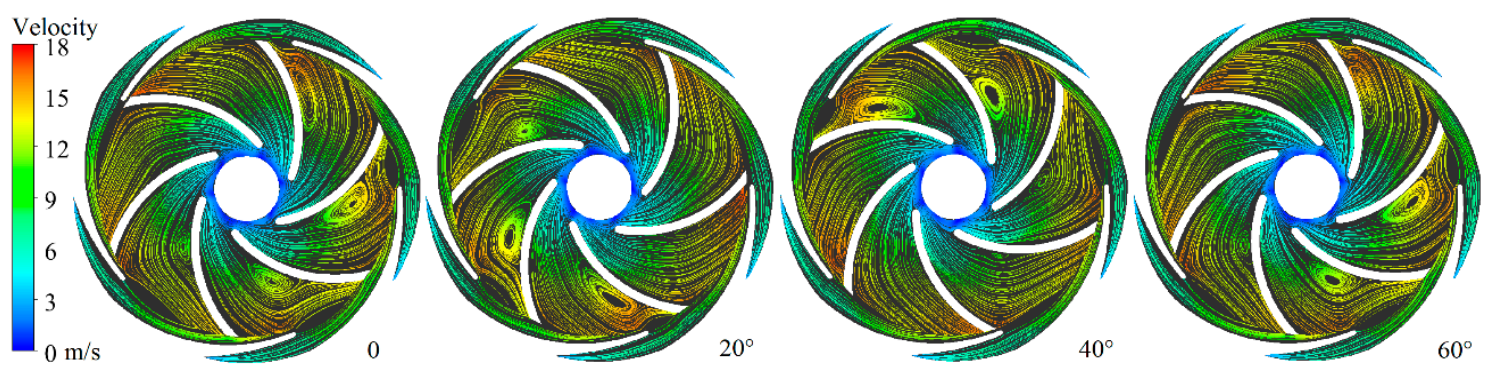

(a) $\beta_{G 1}=5^{\circ}$
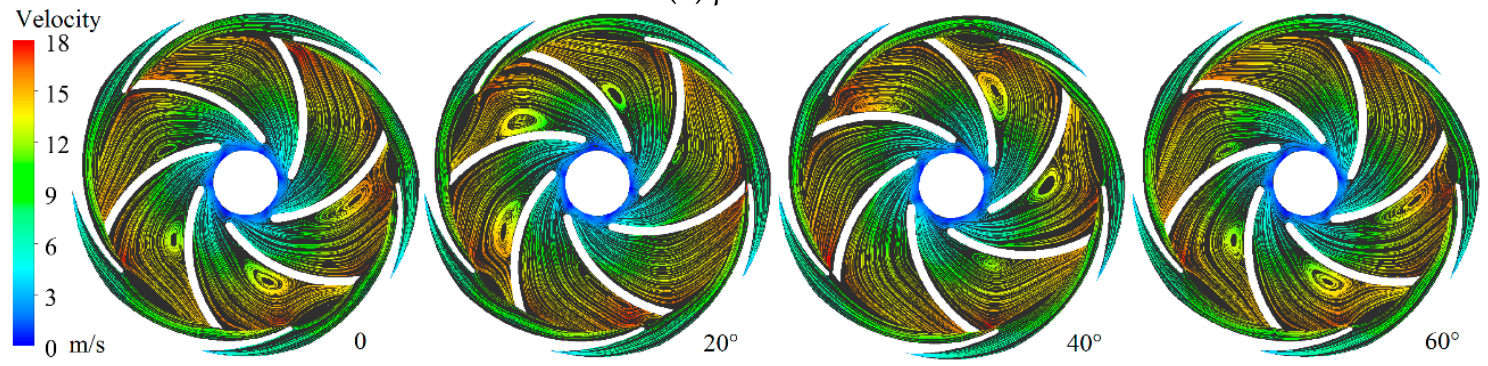

(b) $\beta_{G 1}=10^{\circ}$
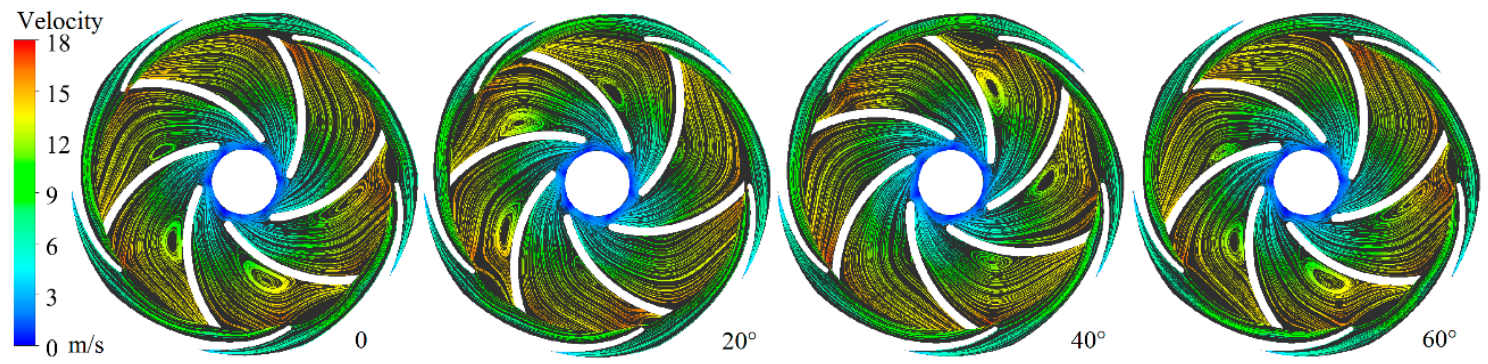

(c) $\beta_{G 1}=15^{\circ}$

Figure 21. Evolution processes of the transient flow field inside the impeller and guide vane with different inlet angles of the positive guide vane.

\subsection{Inlet Diameter of the Guide Vane}

Table 9 shows the correlations between the inlet diameter of the positive guide vane and the hydraulic and hydroacoustic characteristics of the impeller. As the inlet diameter increases, the energy head of the inlet and outlet and the energy increment increase monotonously, whereas the total SPL declines gradually, the shaft power changes are small, and the hydraulic performance of the pump first decreases and then increases.

Table 9. Correlations between the inlet diameter of the positive guide vane and the hydraulic and hydroacoustic characteristics of the impeller.

\begin{tabular}{|c|c|c|c|c|c|c|c|}
\hline \multirow{2}{*}{$\begin{array}{c}\text { Guide Vane } \\
\text { Parameter }\end{array}$} & \multicolumn{5}{|c|}{ Hydraulic and Hydroacoustic Characteristics of the Impeller } & \multicolumn{2}{|c|}{$\begin{array}{l}\text { Hydraulic Performance } \\
\text { of the Pump }\end{array}$} \\
\hline & $\begin{array}{l}\text { Energy head of } \\
\text { inlet/m }\end{array}$ & $\begin{array}{c}\text { Energy head of } \\
\text { outlet/m }\end{array}$ & $\begin{array}{c}\text { Energy } \\
\text { increment } / \mathrm{m}\end{array}$ & $\begin{array}{c}\text { Shaft } \\
\text { power/W }\end{array}$ & $\begin{array}{c}\text { Total } \\
S P L / \mathrm{dBA}\end{array}$ & $\begin{array}{c}\text { Water } \\
\text { head } / \mathrm{m}\end{array}$ & $\begin{array}{l}\text { Hydraulic } \\
\text { efficiency } / \%\end{array}$ \\
\hline 123.2 & 5.89 & 28.85 & 22.96 & 668.5 & 145.3 & 25.06 & 25.82 \\
\hline 125 & 6.51 & 31.09 & 24.58 & 678.1 & 141.6 & 26.85 & 27.27 \\
\hline 127 & 6.39 & 31.56 & 25.17 & 678.2 & 136.2 & 26.75 & 27.17 \\
\hline
\end{tabular}

Figure 22 shows the time-average and the fluctuation intensity curves of pressure and velocity on the blade surface of the impeller with different inlet diameters of the positive guide vane. With the increase in $\mathrm{D}_{\mathrm{G} 1}$, the time-average of pressure and velocity on the blade surface of the impeller change little, the inlet diameter of the positive guide vanes have low impact to the flow of the impeller runner, therefore the divergence of the time-average lines of pressure is small. While their fluctuation intensity 
decreases very dramatically. This is because the increase in the inlet diameter leads to an increase in the clearance between the dynamic and static cascades, and the RSI effect between the guide vane and the impeller is weakened.

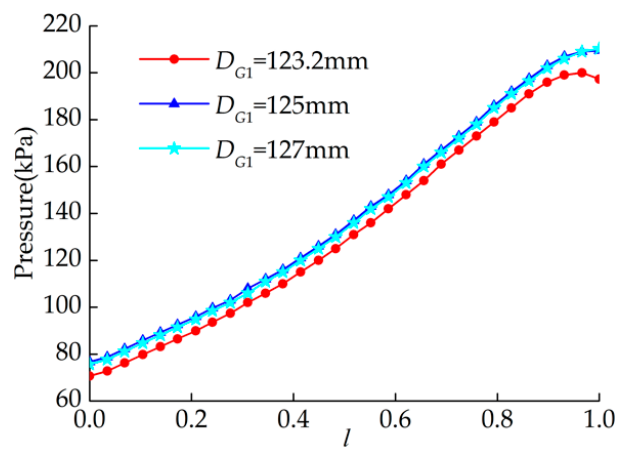

(a)

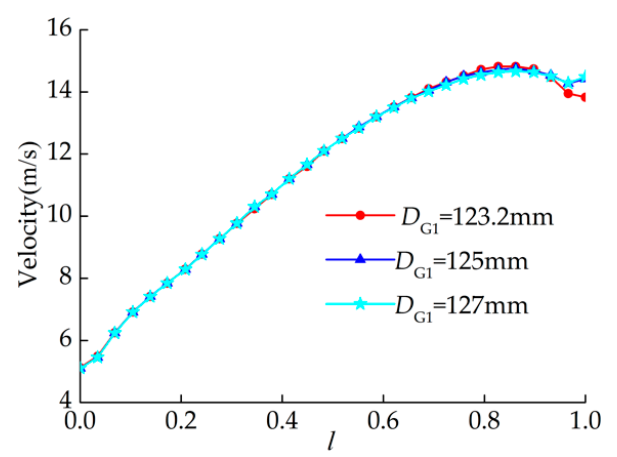

(c)

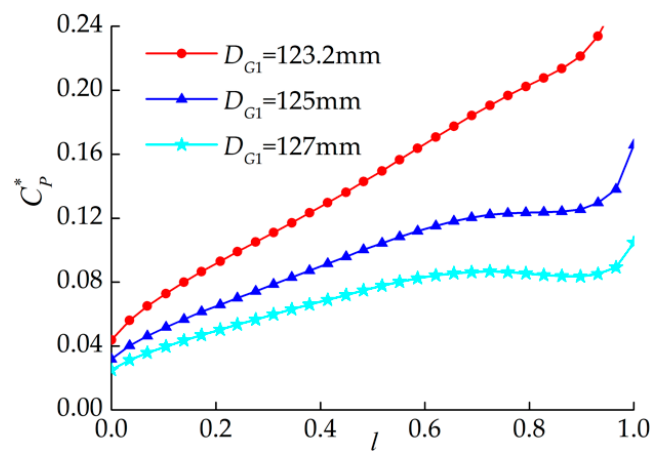

(b)

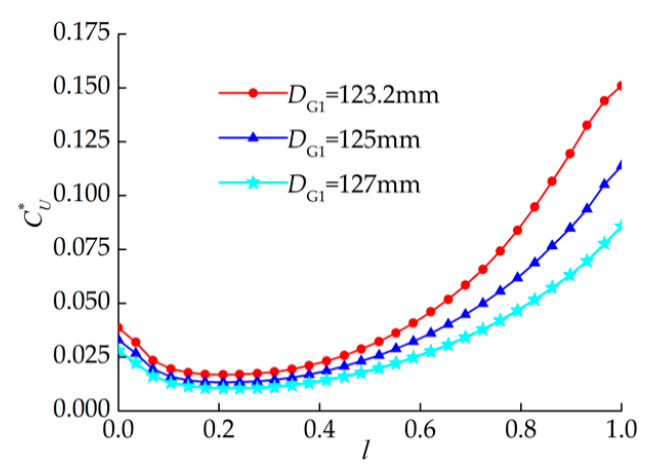

(d)

Figure 22. The time-average and the fluctuation intensity curves of pressure and velocity on the blade surface of the impeller with different inlet diameters of the positive guide vane. (a) The curves of the pressure time-average; (b) the curves of pressure fluctuation intensity; (c) the curves of the velocity time-average; (d) the curves of velocity fluctuation intensity.

Figure 23 shows the time and frequency response curves of the pressure of the impeller with different inlet diameters of the positive guide vane. The variation rules of the pressure in the time and frequency domains are highly similar for different $D_{G 1}$. With the increase in $D_{G 1}$, the RSI effect is weakened, and the fluctuation amplitudes decrease gradually at major characteristic frequencies.

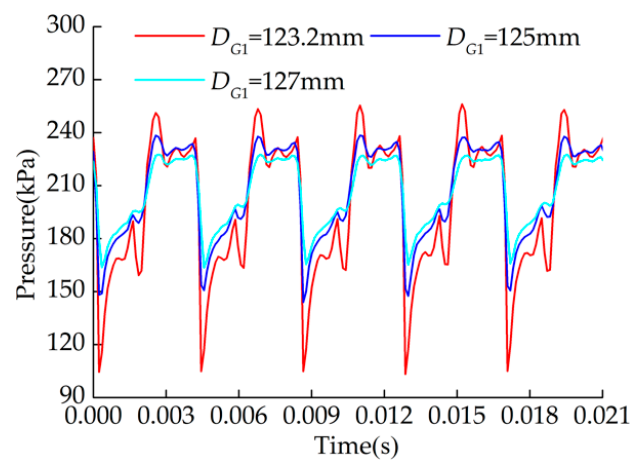

(a) Time domain

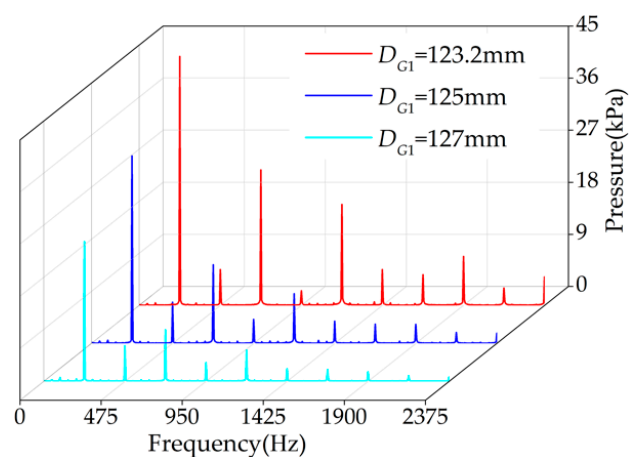

(b) Frequency domain

Figure 23. Frequency response curves of pressure inside the impeller with different inlet diameters of the positive guide vane.

Figure 24 shows the frequency response curves of the hydroacoustic SPL of the impeller with different inlet diameters of the positive guide vane. The frequency spectrum characteristic of the SPL is 
similar for different inlet diameters; the dominant frequencies of all of them are $B P F_{I}$, and the SPL decreases as the inlet diameter increases.

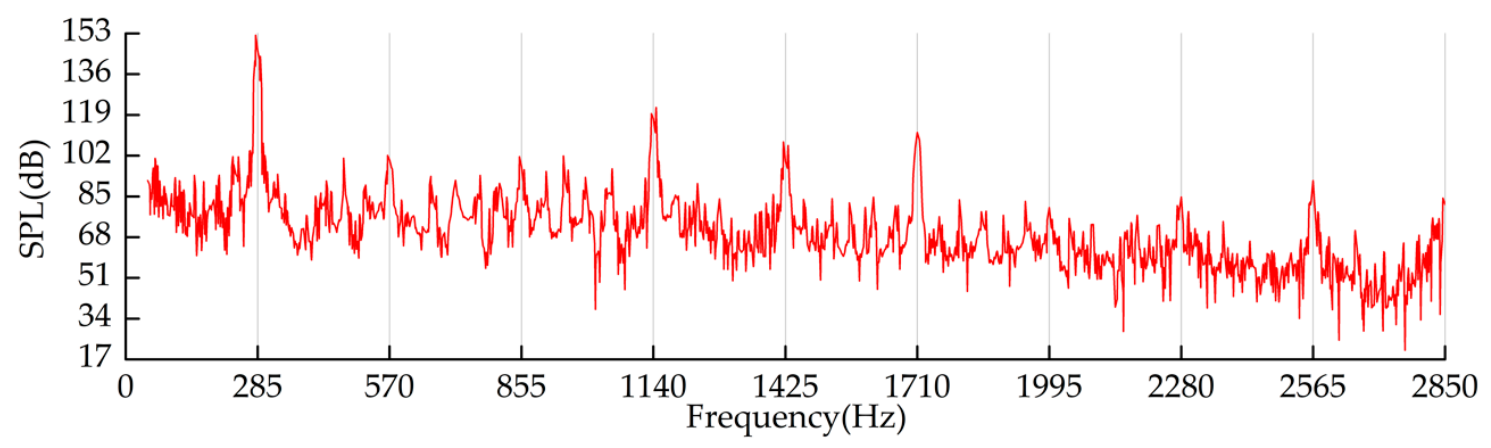

(a) $D_{G 1}=123.2 \mathrm{~mm}$

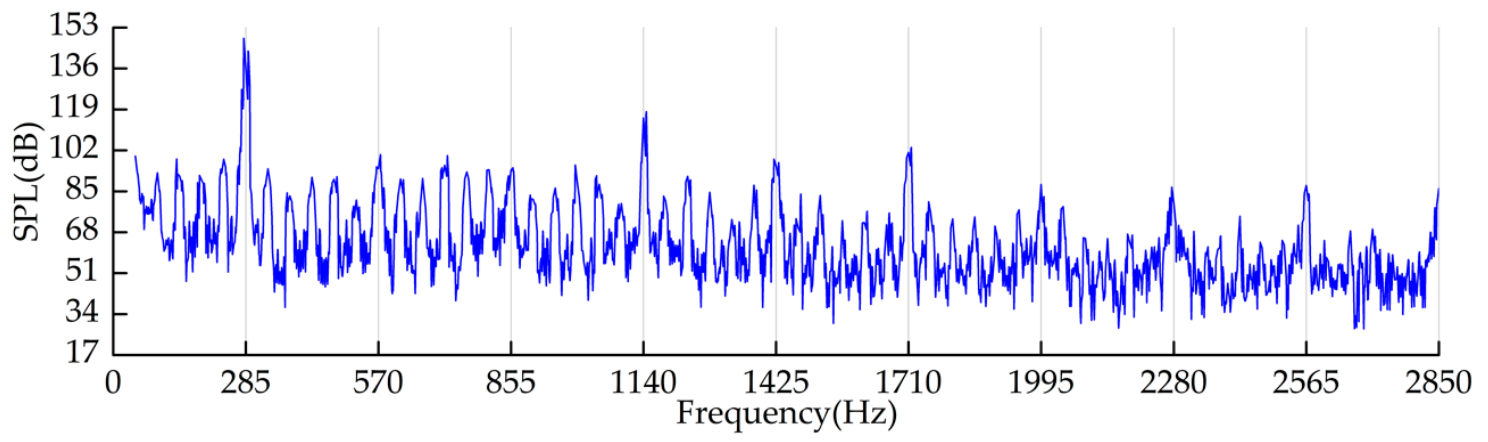

(b) $D_{G 1}=125 \mathrm{~mm}$

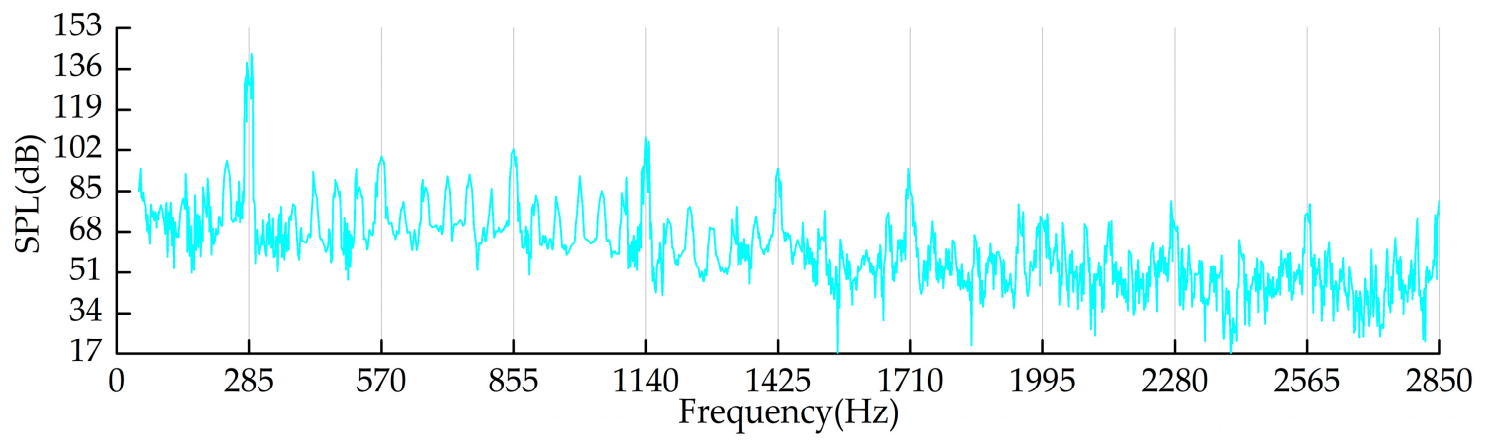

(c) $D_{\mathrm{G} 1}=127 \mathrm{~mm}$

Figure 24. Frequency response curves of the hydroacoustic SPL inside the impeller with different inlet diameters of the positive guide vane.

Figure 25 shows the evolution processes of the flow field inside the impeller and guide vane with different inlet diameters of the positive guide vane, and the evolution rules of the flow field are basically the same in the dynamic and static cascades. With the increase in $D_{G 1}$, the size and intensity of vortices inside the impeller and the degree of flow blockage at the inlet of the guide vane decrease gradually, and the reasons are the same as those that drive the variation in the fluctuation intensity of the flow field on the blade surface. 

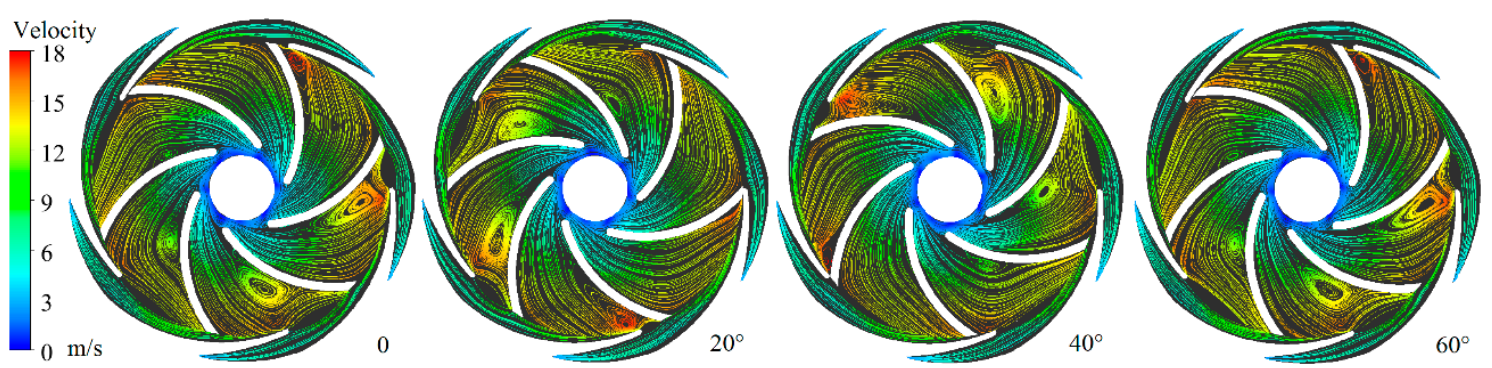

(a) $D_{G 1}=123.2 \mathrm{~mm}$
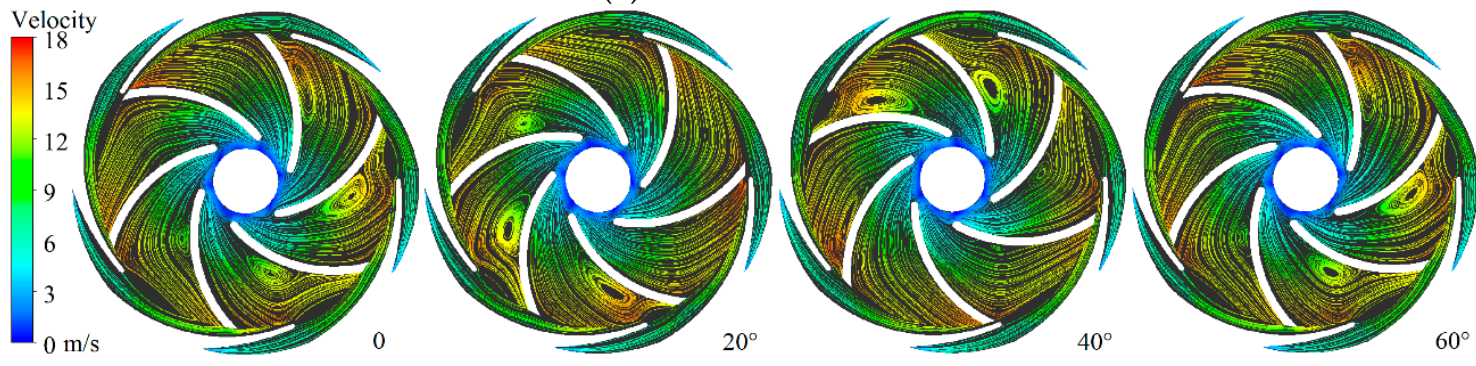

(b) $D_{G 1}=125 \mathrm{~mm}$
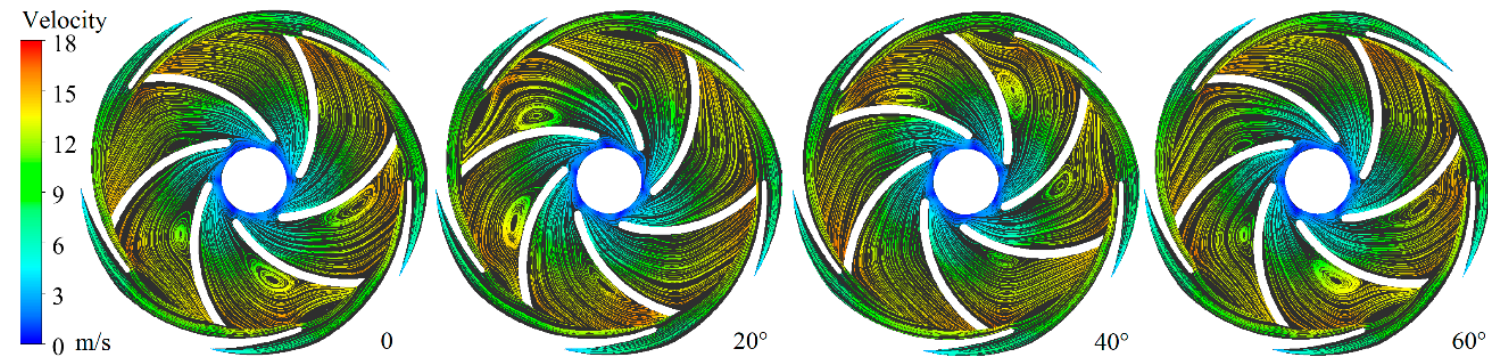

(c) $D_{G 1}=127 \mathrm{~mm}$

Figure 25. Evolution processes of the transient flow field inside the impeller and guide vane with different inlet diameters of the positive guide vane.

The analyses in Sections 3.1-3.5 show that a good guide vane can improve the transient flow field structure inside the impeller, reduce the flow field distortion phenomena, and improve the hydroacoustic characteristic of the impeller and the hydraulic performance of the JCP. When the impeller geometry is constant, the evolution process of the flow field inside the impeller is mainly related to the blade number of the guide vane, and other parameters only affect the strength and size of the vortices without changing their basic law. When the number of guide vanes is given, the RSI effect on the hydroacoustic characteristic of the impeller is characterized by a positive correlation between the total SPL and the fluctuation intensity of the flow field.

\section{Optimization Design of the Guide Vane}

The total SPL of the impeller was taken as the objective to optimize the effects of the rotor-stator interaction on the hydraulic/hydroacoustic characteristics of the JCP impeller, and an orthogonal experimental method was used to optimize the structural parameters of the guide vane for the stability of the hydraulic performance of the JCP.

\subsection{The Scheme and Results of the Orthogonal Experiment}

The L9 $\left(3^{4}\right)$ standard orthogonal table [21] was used to construct the experimental design of the structural parameters of the guide vane: with the three factor levels discussed in Section 3 of the paper, nine schemes of the guide vane structure were obtained, and then their flow field and sound field were calculated. Table 10 specifies the levels of the orthogonal experimental factors, and $A, B, C$, and $D$ 
are factor codes that correspond to $Z_{2}, \varphi_{2}, \beta_{G 1}$, and $D_{G 1}$, and Table 11 is the experimental schemes and results.

Table 10. Levels of the orthogonal experimental factors.

\begin{tabular}{ccccc}
\hline \multirow{2}{*}{ Level } & \multicolumn{5}{c}{ Factors } \\
\cline { 2 - 5 } & $A / \mathbf{Z}_{\mathbf{2}}$ & $\boldsymbol{B} / \boldsymbol{\varphi}_{\mathbf{2}}$ & $\boldsymbol{C} / \boldsymbol{\beta}_{\boldsymbol{G} \mathbf{1}}$ & $\boldsymbol{D} / \boldsymbol{D}_{\boldsymbol{G} \mathbf{1}}$ \\
\hline 1 & 3 & $52^{\circ}$ & $5^{\circ}$ & $123.2 \mathrm{~mm}$ \\
2 & 5 & $68^{\circ}$ & $10^{\circ}$ & $125 \mathrm{~mm}$ \\
3 & 7 & $84^{\circ}$ & $15^{\circ}$ & $127 \mathrm{~mm}$ \\
\hline
\end{tabular}

Table 11. Orthogonal experimental schemes and results.

\begin{tabular}{|c|c|c|c|c|c|c|c|}
\hline Number & $A / Z_{2}$ & $B / \varphi_{2}$ & $C / \beta_{G 1}$ & $D / D_{G 1}$ & Total SPL of Impeller/ $L_{P}(\mathrm{dBA})$ & Water Head/H (m) & Hydraulic Efficiency $/ \eta$ (\%) \\
\hline 1 & 1 & 1 & 1 & 1 & 143.2 & 15.99 & 18.94 \\
\hline 2 & 1 & 2 & 2 & 2 & 140.0 & 20.10 & 21.69 \\
\hline 3 & 1 & 3 & 3 & 3 & 134.6 & 16.33 & 19.20 \\
\hline 4 & 2 & 2 & 1 & 3 & 136.2 & 26.42 & 27.16 \\
\hline 5 & 2 & 3 & 2 & 1 & 143.9 & 23.66 & 24.05 \\
\hline 6 & 2 & 1 & 3 & 2 & 139.1 & 24.28 & 25.06 \\
\hline 9 & 3 & 2 & 3 & 1 & 142.1 & 23.25 & 23.79 \\
\hline
\end{tabular}

\subsection{The Intuitionistic Analysis}

The range of values reflects the influence degree of the change in the factor level on the index. The greater the range, the greater the influence of the selected factor on the index. Therefore, the most significant factor corresponds to the largest range. Because of the positive correlation between the head and efficiency, the hydraulic performance of the pump only reflects efficiency. A range analysis was carried out on the numerical calculation results in Table 10. Range analysis was carried out by Equations (12) and (13), where $k_{i}$ is the average value of $j$ levels of factor $i$; $s$ is range.

$$
\begin{gathered}
k_{i}=\frac{1}{N_{i}} \sum_{j=1}^{N_{i}} K_{i, j}, \\
s=\max \left(k_{1}, k_{2}, \cdots, k_{i}\right)-\min \left(k_{1}, k_{2}, \cdots, k_{i}\right)
\end{gathered}
$$

The results are shown in Table 12. As shown in the table, the sequence of the influence degree of the guide vane parameters on the hydraulic efficiency of the pump is $A, D, C, B$, and the sequence of the influence degree of the same parameters on the hydroacoustic performance of the impeller is $D, C$, $B, A$.

Table 12. Results of range analysis.

\begin{tabular}{ccccccccc}
\hline & \multicolumn{3}{c}{ Total $S P L$ of the Impeller/ $\boldsymbol{L}_{\boldsymbol{P}} \mathbf{( d B A )}$} & \multicolumn{3}{c}{ Hydraulic Efficiency of the Pump/ $\boldsymbol{\eta}(\%)$} \\
\hline Factor & $k_{1}$ & $k_{2}$ & $k_{3}$ & $s$ & $k_{1}$ & $k_{2}$ & $k_{3}$ & $s$ \\
\hline$A$ & 139.3 & 139.7 & 139.9 & 0.60 & 19.94 & 25.42 & 24.84 & 5.48 \\
$B$ & 140.9 & 139.4 & 138.6 & 2.30 & 23.29 & 24.21 & 22.70 & 1.20 \\
$C$ & 138.9 & 141.4 & 138.6 & 2.80 & 23.65 & 23.88 & 22.68 & 1.51 \\
D & 143.1 & 138.8 & 137.0 & 6.03 & 22.26 & 23.86 & 24.08 & 1.82 \\
\hline
\end{tabular}

Figure 26 presents the effective curves of the optimization indexes. For the hydroacoustic performance of the impeller, the smaller the index, the better the performance; therefore, the relative optimal parameter combination is $A_{1} B_{3} C_{3} D_{3}$. For hydraulic efficiency, the higher the index, the better the performance, so the relative optimal combination is $A_{2} B_{2} C_{2} D_{3}$. 


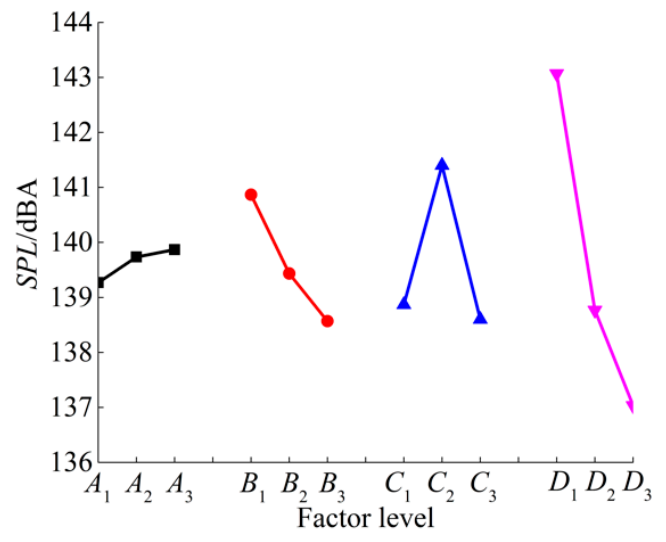

(a) Hydroacoustic performance of the impeller

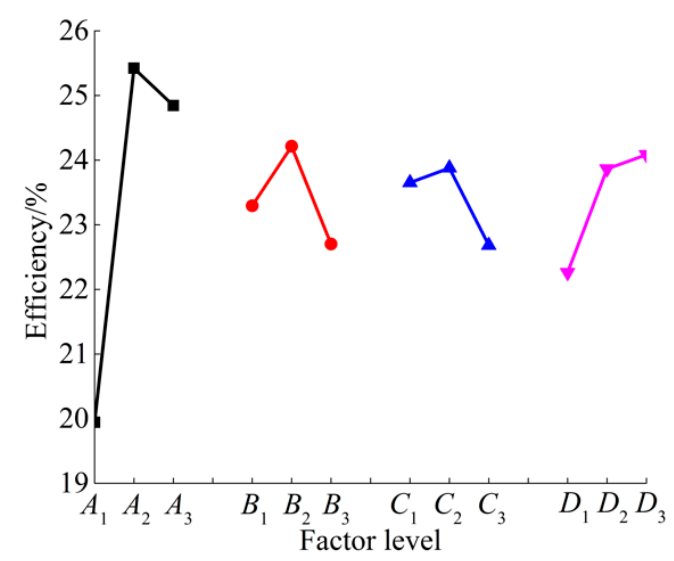

(b) Hydraulic efficiency of the pump

Figure 26. Effective curves of optimization indexes.

\subsection{The Global Optimization Scheme}

The matrix analysis method described in Section 2.6 was employed to obtain the global optimization scheme for both the hydraulic efficiency of the pump and hydroacoustic performance of the impeller. The weight matrices corresponding to hydraulic efficiency and the total SPL were calculated by Equations (7)-(11). For hydraulic efficiency, $K_{i j}=k_{i j}$; for the hydroacoustic SPL, $K_{i j}=1 / k_{i j}$. The results are shown in Equations (14) and (15), and Equation (16) is the global matrix, which is the average of Equations (14) and (15).

$$
\begin{aligned}
\omega_{\eta} & =(15.54,19.81,19.36,5.06,5.21,4.89,4.03,4.07,3.86,5.77,6.19,6.25)^{\mathrm{T}} \times 10^{-2} \\
\omega_{L P} & =(1.71,1.70,1.70,6.45,6.542,6.58,7.80,7.85,8.01,16.73,17.24,17.46)^{\mathrm{T}} \times 10^{-2} \\
\omega_{\text {avg }} & =(8.63,10.76,10.53,5.75,5.89,5.74,6.01,5.96,5.94,11.25,11.75,11.85)^{\mathrm{T}} \times 10^{-2}
\end{aligned}
$$

These results show that the primary and secondary order of influence of different factors on the global performance is $D, A, C, B$. Factors $A$ and $B$ are optimal at level 2, factor $C$ is optimal at level 1, and factor $D$ is optimal at level 3. Therefore, the global optimal scheme is $A_{2} B_{2} C_{1} D_{3}$ : that is, the number of blades $Z_{2}=5$, wrap angle $\varphi_{2}=68^{\circ}$, inlet angle $\beta_{G 1}=5^{\circ}$, and inlet diameter $D_{G 1}=127 \mathrm{~mm}$.

Figure 27 compares the performance of the prototype and that of the optimization scheme. After optimization, the hydraulic performance of the pump is stable under all conditions, and the hydroacoustic performance of the impeller is significantly reduced, so the desired objectives are realized.

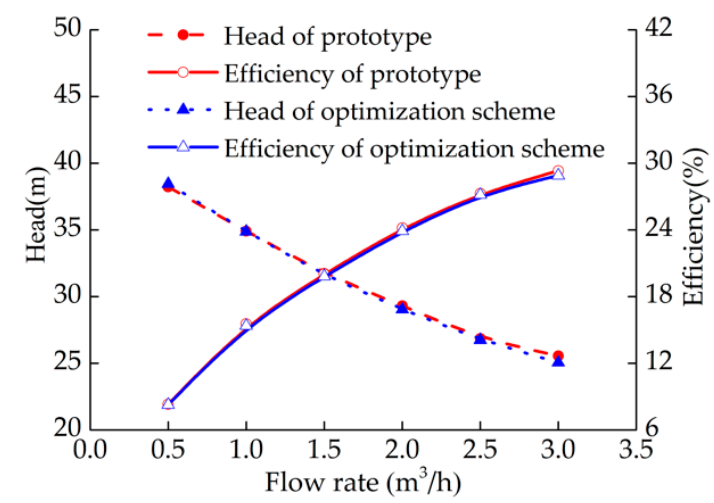

(a) Hydraulic performance of the JCP

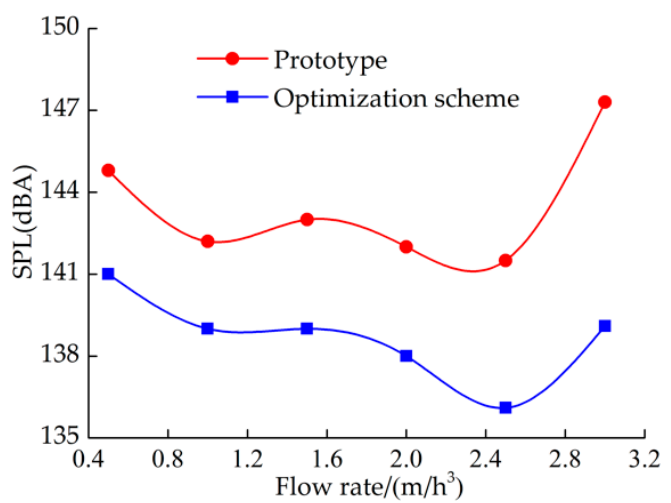

(b) Hydroacoustic performance of the impeller

Figure 27. Performance comparison between the prototype and optimization scheme. 


\section{Conclusions}

In this paper, a numerical method that couples CFD/CFA was used to analyze the correlation between the guide vanes and the hydraulic/hydroacoustic characteristics of an impeller. In addition, the hydroacoustic performance of the impeller was taken as the objective to optimize the structural parameters of the guide vane for the stability of the hydraulic performance of the JCP using the orthogonal test method. Several conclusions are drawn from the results:

(1) The RSI leads to a significant increase in the hydroacoustic level of the impeller, but it is indispensable for improving the hydraulic performance of the pump; a good guide vane can improve the transient flow field structure inside the impeller, reduce the flow field distortion phenomena, and improve the hydraulic and hydroacoustic performance of the impeller.

(2) The fluctuation intensity of the transient flow field inside the impeller is much more sensitive than the time-average to the RSI effect; inside the impeller, it has a positive correlation between the fluctuation intensity of the flow field and the vortex intensity.

(3) When the impeller geometry is constant, the evolution processes of the flow field inside the impeller are mainly related to the blade number of the guide vane, and other structural parameters only affect the strength and size of vortices without changing their basic law. When the number of guide vanes is given, the RSI effect on the hydroacoustic characteristic of the impeller is characterized by a positive correlation between the total SPL and the fluctuation intensity of the flow field.

(4) The frequency spectrum characteristics of the hydroacoustic SPL of the impeller are related not only to the blade number and speed of the impeller but also to its wake characteristics determined by guide vane. Moreover, the frequency spectrum characteristics of the hydroacoustic SPL are not consistent with the pressure fluctuation characteristics inside the impeller.

(5) Under the design conditions, the optimization scheme for the stable hydraulic performance of the JCP significantly reduces the total SPL of the impeller compared with the original scheme, which verifies the feasibility of using the weight matrix optimization method to obtain the global optimization scheme.

Author Contributions: Formal analysis, R.G.; funding acquisition, R.L.; methodology, R.G.; resources, R.Z.; validation, W.H.; writing — original draft preparation, R.G.; writing-review and editing, R.L. All authors have read and agreed to the published version of the manuscript.

Funding: This research was funded by the Natural Science Foundation of China, grant number 51579125, and the National Key Research and Development Program of China, grant number 2016YFB0200901.

Acknowledgments: The comments of two anonymous reviewers, and the help of the Academic Editor and the Assistant Editor are highly appreciated.

Conflicts of Interest: The authors declare no conflicts of interest.

\section{References}

1. Shirani, E.; Daneshkhah, K. Rotor-stator interaction in turbomachines. J. Appl Sci. 2002, 2, 955-964. [CrossRef]

2. Liu, P.; Chen, X.L.; Wang, Q.; Li, D.Z. Analysis of rotor-stator interaction and vibration in high-head Francis turbines. J. Hydroelectr. Eng. 2016, 35, 91-98. [CrossRef]

3. Wang, T.; Gu, C.G. Numerical simulation of rotor/stator interaction in centrifugal fan at designed condition. Chin. J. Mech. Eng. 2001, 37, 20-24. [CrossRef]

4. Dong, R.; Chu, S.; Katz, J. Effect of modification to tongue and impeller geometry on unsteady flow, pressure fluctuations, and noise in a centrifugal pump. J. Turbomach. 1997, 119, 506-515. [CrossRef]

5. González, J.; Fernández, J.; Blanco, E.; Santolaria, C. Numerical simulation of the dynamic effects due to impeller-volute interaction in a centrifugal pump. J. Fluids Eng. 2002, 124, 348-355. [CrossRef]

6. Guo, S.J.; Okamoto, H.; Maruta, Y. Measurement on the fluid forces induced by rotor-stator interaction in a centrifugal pump. JSME Int. J. Ser. B 2006, 49, 434-442. [CrossRef] 
7. Rodriguez, C.; Egusquiza, E.; Santos, I. Frequencies in the vibration induced by the rotor stator interaction in a centrifugal pump turbine. J. Fluids Eng. 2007, 129, 1428-1435. [CrossRef]

8. Nicolet, C.; Ruchonnet, N.; Alligné, S.; Koutnik, J.; Avellan, F. Hydroacoustic simulation of rotor-stator interaction in resonance conditions in Francis pump-turbine. In IOP Conference Series: Earth and Environmental Science; IOP Publishing: Bristol, UK, 2010; Volume 12, p. 012005. [CrossRef]

9. Zhu, L.; Yuan, S.Q.; Yuan, J.P.; Pei, J. Numerical simulation on rotor-stator interaction in a centrifugal pump with different gaps between impeller and tongue. Trans. Chin. Soc. Agric. Mach. 2011, 42, 49-55. [CrossRef]

10. Zhu, L.; Yuan, S.Q.; Yuan, J.P.; Zhou, J.Q.; Jin, R.; Wang, H. Numerical simulation for rotor-stator interaction of centrifugal pump with different tongues. Trans. Chin. Soc. Agric. Mach. 2011, 27, 50-55. [CrossRef]

11. Rodriguez, C.G.; Borja, M.P.; Eduard, E. Monitoring of rotor-stator interaction in pump-turbine using vibrations measured with on-board sensors rotating with shaft. Shock Vib. 2014, 2014, 1-8. [CrossRef]

12. Zhou, P.J.; Wang, F.J.; Yao, Z.F. Impeller-volute interaction around tongue region in centrifugal pump under rotating stall condition. Trans. Chin. Soc. Agric. Eng. 2015, 31, 85-90. [CrossRef]

13. Zhang, X.; Wang, P.F.; Ruan, X.D.; Xu, Z.B.; Fu, X. Analysis of pressure pulsation induced by rotor-Stator interaction in nuclear reactor coolant pump. Shock Vib. 2017, 2017, 1-18. [CrossRef]

14. Chalghoum, I.; Kanfoudi, H.; Elaoud, S.; Akrout, M.; Zgolli, R. Numerical modeling of the flow inside a centrifugal pump: Influence of impeller-volute interaction on velocity and pressure fields. Arab. J. Sci. Eng. 2016, 41, 4463-4476. [CrossRef]

15. Chalghoum, I.; Elaoud, S.; Kanfoudi, H.; Akrout, M. The effects of the rotor-stator interaction on unsteady pressure pulsation and radial force in a centrifugal pump. J. Hydrol. 2018, 30, 136-145. [CrossRef]

16. Celik, I.B.; Ghia, U.; Roache, P.J.; Freitas, C.J.; Coleman, H.; Raad, P.E. Procedure for estimation and reporting of uncertainty due to discretization in CFD applications. J. Fluids Eng. 2008, 130, 078001-078004. [CrossRef]

17. Ansys Inc. Ansys Fluent Theory Guide; Ansys Inc.: Canonsburg, PA, USA, 2015.

18. Pei, J.; Yuan, S.Q.; Yuan, J.P.; Wang, W.J. Comparative study of pressure fluctuation intensity for a single blade pump under multiple operating conditions. J. Huazhong Univ. Sci. Technol. 2013, 41, 29-33. [CrossRef]

19. Wang, F.; Wang, J.C. Noise measurement and calculation of the relationship between one-third octave and octave spectrum. Noise Vib. Control 1996, 5, 39-41.

20. Wei, X.L.; Xue, B.J.; Zhao, Q. Optimization design of the stability for the plunger assembly of oil pumps based on multi-target orthogonal test design [J]. J. Hebei Univ. Eng. (Nat. Sci. Ed.) 2010, 27, 95-99.

21. Zhao, X.M. Test Design Method [M]; Science Press: Beijing, China, 2010. (In Chinese) 